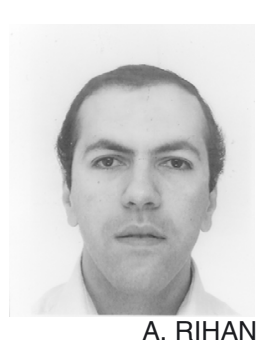

\title{
Réalisation d'un oscillateur paramétrique optique continûment accordable dans l'infrarouge pour des applications radiométrique et pyrométrique
}

\section{Development of a continuously tunable mid-infrared optical parametric oscillator for radiometric and pyrometric applications}

\author{
Abdallah RIHAN, Emeline ANDRIEUX, Thomas ZANON-WILLETTE, Malo CADORET et \\ Jean-Jacques ZONDY
}

Laboratoire commun de métrologie LNE-CNAM (LCM), CNAM, 61 Rue du Landy, 93210 La Plaine Saint Denis, France, abdallah.rihan@cnam.fr.

\section{Résumé}

Nous présentons dans cet article un oscillateur paramétrique optique résonant sur la pompe et le signal (PRSRO), largement et continûment accordable, délivrant $20 \mathrm{~mW}$ à $50 \mathrm{~mW}$ de puissance dans l'onde complémentaire (idler) couvrant une octave de longueur d'onde dans le moyen infrarouge $(1,7 \mu \mathrm{m}$ à $3,5 \mu \mathrm{m})$. Ce PRSRO est pompé par un laser $\mathrm{Ti}: \mathrm{Al}_{2} \mathrm{O}_{3}$ en anneau mono-fréquence et accordable, délivrant $760 \mathrm{~mW}$ autour de $795 \mathrm{~nm}$, et grâce à la résonnance de la pompe dans la cavité OPO, un seuil d'oscillation de $110 \mathrm{~mW}$ est atteint. Cette source laser non-linéaire largement accordable dans l'IR est un des maillons de la chaîne laser radiométrique devant permettre de couvrir le spectre électromagnétique de l'UV à l'IR moyen, pour le raccordement de la sensibilité spectrale des détecteurs au moyen du radiomètre cryogénique. Cette source est également utilisable pour la spectroscopie moléculaire haute résolution dans le domaine spectrale $1 \mu \mathrm{m}-3,5 \mu \mathrm{m}$.

MOTS CLÉS : OPTIQUE NON LINÉAIRE, OSCILLATEUR PARAMÉTRIQUE OPTIQUE, OPTIQUE PARAMETRIQUE, QUASIACCORD DE PHASE, SOURCE LASER ACCORDABLE, INFRAROUGE, ASSERVISSEMENT EN FRÉQUENCE.

\footnotetext{
Abstract

We report on a widely tunable continuous-wave single-frequency pump-resonant signal-resonant optical parametric oscillator (PRSRO) delivering $20 \mathrm{~mW}$ to $50 \mathrm{~mW}$ of idler power spanning an octave bandwidth in the mid-infrared $(1.7 \mu \mathrm{m}-3.5 \mu \mathrm{m})$. This PRSRO is pumped by a continuous-wave $\mathrm{Ti}: \mathrm{Al}_{2} \mathrm{O}_{3}$ single-frequency and tunable laser delivering $760 \mathrm{~mW}$ around $795 \mathrm{~nm}$, and thanks to the pump enhancement a
}

threshold as low as $110 \mathrm{~mW}$ is achieved. This nonlinear laser source which is widely tunable in the mid-IR spectral range is part of the radiometric laser chain project aimed at the calibration of the spectral responsivity of photo-detectors in the UV-IR range by spectral irradiance measurement via a cryogenic radiometer. This widely tunable source is also suited to high-resolution molecular spectroscopy in the mid-infrared range.

KEYWORDS: NONLINEAR OPTICS, OPTICAL PARAMETRIC OSCILLATOR, PARAMETRIC INTERACTIONS, QUASI-PHASEMATCHING, TUNABLE LASER, INFRARED, SERVO-CONTROL.

\section{Introduction}

Le radiomètre cryogénique est la référence primaire pour l'étalonnage en sensibilité spectrale des détecteurs. Pour pouvoir utiliser pleinement les performances de cet appareil, il est nécessaire de disposer de faisceaux lasers couvrant un domaine spectral aussi large que possible et ayant des caractéristiques géométriques adaptées. Actuellement, les étalonnages des détecteurs par comparaison directe au radiomètre cryogénique ne sont réalisés qu'à certaines longueurs d'onde laser discrètes du domaine visible, entre $454 \mathrm{~nm}$ (laser argon) et $633 \mathrm{~nm}$ (laser $\mathrm{He}-\mathrm{Ne}$ ), avec une incertitude de l'ordre de $10^{-4}$. Les valeurs à d'autres longueurs d'onde, dans le domaine $200 \mathrm{~nm}-2000 \mathrm{~nm}$, sont obtenues au LCM par 
diverses méthodes d'interpolation ou d'extrapolation qui conduisent à une dégradation importante de l'incertitude. Dans ces conditions, le raccordement au radiomètre cryogénique des détecteurs de transfert n'est réalisé qu'avec une incertitude supérieure à $10^{-3}$. Ainsi avec une source continûment accordable sur un domaine spectral de $200 \mathrm{~nm}$ à $4000 \mathrm{~nm}$, la sensibilité spectrale des détecteurs pourra être mesurée directement par rapport au radiomètre cryogénique à n'importe quelle longueur d'onde en conservant l'incertitude de l'ordre de $10^{-4}$, déjà obtenue jusqu'alors entre $454 \mathrm{~nm}$ et $633 \mathrm{~nm}$. De plus, le raccordement au radiomètre cryogénique des détecteurs de transfert pourra être étendu à d'autres types de détecteurs que les photodiodes au silicium (domaine spectral $350 \mathrm{~nm}-1100 \mathrm{~nm}$ ), pour couvrir des domaines ultraviolet et infrarouge où, pour le moment, les incertitudes sont trop élevées pour répondre correctement aux besoins.

De plus, en radiométrie des sources, les références d'éclairement énergétique sont faites par des radiomètres à filtre dont la sensibilité est étalonnée. Une source laser accordable associée à un détecteur de référence permet l'étalonnage de ce radiomètre plus précisément que par la méthode actuelle utilisant une source monochromatique non cohérente. La bonne connaissance de la longueur d'onde d'un faisceau laser est un élément important de cette amélioration mais l'étalonnage de la référence de sensibilité directement par rapport au radiomètre cryogénique donne accès à des incertitudes de l'ordre de $10^{-4}$. Un détecteur étalonné à ce niveau d'incertitude apportera donc une amélioration d'un facteur 10 par rapport au niveau d'incertitude actuel.

Par ailleurs en pyrométrie, la mesure de la température passe par la mesure de la luminance. Un faisceau laser est à la base de la constitution d'une source monochromatique de surface étendue $(5 \mathrm{~mm})$ dont la luminance est mesurée en connaissant l'étendue géométrique et en disposant d'un détecteur étalonné en sensibilité spectrale. Une telle source étalon de luminance permet alors de connaître la sensibilité spectrale absolue d'un pyromètre ou d'un luminance-mètre. En couplant de façon rapprochée l'étalonnage d'un pyromètre et la mesure du rayonnement issu d'un corps noir, on en déduit sa température thermody-namique. L'objectif de quelques dizaines de millikelvins à une centaine de millikelvins dans le domaine de température entre $1000{ }^{\circ} \mathrm{C}$ et $3000^{\circ} \mathrm{C}$ peut être obtenu grâce aux performances de la source laser accordable et des étalonnages réalisés vis-à-vis du radiomètre cryogénique.

Pour la couverture spectrale dans le domaine infrarouge (IR), plusieurs sources lasers sont possibles. Les lasers à cascades quantiques (QCL) couvrent le domaine spectrale s'étendant du moyen infrarouge à partir de 3,5 $\mu \mathrm{m}$ au domaine du térahertz, néanmoins leurs accordabilité en régime continu est de quelques centaines de nanomètres en cavité étendue [1]. Ils ne peuvent donc pas rivaliser avec la très grande accordabilité des oscillateurs paramétriques optiques (OPO) [2-5] et sont par ailleurs encore très onéreux. Une autre alternative serait de faire de la différence de fréquences (DFG) de deux sources lasers dans le visible/proche-IR, qui est un dispositif plus simple à mettre en œuvre qu'un OPO, mais qui nécessite deux lasers pompes puissants et dont l'un des deux au moins accordable. Un tel dispositif ne permet d'avoir par ailleurs qu'une puissance de quelques nanowatts à dizaine de microwatts, sauf si les deux lasers ont une puissance supérieure à $5 \mathrm{~W}$ [6]. Pour augmenter le niveau de puissance de l'onde ainsi générée, il est nécessaire d'utiliser une cavité de surtension sur la pompe [7], ce qui conduit à un degré de complexité similaire à celui d'un OPO qui n'utilise qu'un seul laser de pompe.

Cet article est organisé en cinq parties. La partie 2 rappelle brièvement le principe de fonctionnement d'un oscillateur paramétrique optique. La partie 3 traite du quasi-accord de phase (QPM) du $\mathrm{LiNbO}_{3}$ à inversion de domaines de polarisation utilisé comme cristal non linéaire pompé à une longueur d'onde $\lambda_{\mathrm{p}}=795 \mathrm{~nm}$. La partie 4 présente le dispositif expérimental et décrit l'asservissement électronique de la cavité OPO. La partie 5 présente et analyse les performances du PRSRO en termes de puissance et d'accordabilité spectrale. Et nous concluons avec la partie 6 où nous donnons les perspectives d'améliorations des performances actuelles en termes de puissances idler et de couverture spectrale dans le moyen infrarouge.

\section{Oscillateur paramétrique optique : principe}

Un OPO est un oscillateur laser où le gain ne dépend pas d'une inversion de population atomique ou moléculaire entre deux niveaux d'énergie, mais d'une condition d'accord de phase (phase-matching) liant l'onde «pompe» (de fréquence angulaire $\omega_{\mathrm{p}}$ ) et les deux ondes paramétriquement générées (onde «signal » de fréquence $\omega_{\mathrm{s}}$ et "complémentaire » ou idler de fréquence $\omega_{\mathrm{i}}$ ) par le couplage non linéaire $\chi^{(2)}$ (susceptibilité non linéaire d'ordre 2) du matériau de gain (fig. 1ab) [8]. Dans une conversion paramétrique de fréquence, la conservation de l'énergie du photon pompe est respectée $\left(\hbar \omega_{\mathrm{p}}=\hbar \omega_{\mathrm{s}}+\hbar \omega_{\mathrm{i}}\right.$, fig. 1d). La condition d'accord de phase qui stipule que l'impulsion du photon pompe doit être égale à la somme des impulsions des photons générés $\left(\hbar \mathbf{k}_{\mathrm{p}}=\hbar \mathbf{k}_{\mathrm{s}}+\hbar \mathbf{k}_{\mathrm{i}}\right.$, Fig. 1c), dépend en particulier des propriétés de dispersion spectrale du matériau non-linéaire biréfringent.

Depuis les années 1990, de nouveaux matériaux ferro-électriques micro-structurés par inversion périodique de leur domaines de polarisation spontanée (quasiphase-matching ou QPM) ont émergé, permettant un accord de phase à la carte pour générer les deux ondes paramétriques dans toute la fenêtre de transparence du matériau $[9,10]$. Contrairement à l'accord de phase par biréfringence (birefringent phase-matching ou BPM) qui utilise les deux nappes de l'ellipsoïde des indices pour satisfaire $\Delta \mathbf{k}=0$, le quasi-phasematching peut être réalisé même dans des matériaux non biréfringents pour lesquels la condition $\Delta \mathbf{k}=\mathbf{k}_{\mathrm{p}}-\mathbf{k}_{\mathrm{s}}-\mathbf{k}_{\mathrm{i}}=0$ - qui devient scalaire lorsque l'accord de phase est colinéaire ne peut être réalisée à cause de la dispersion normale 


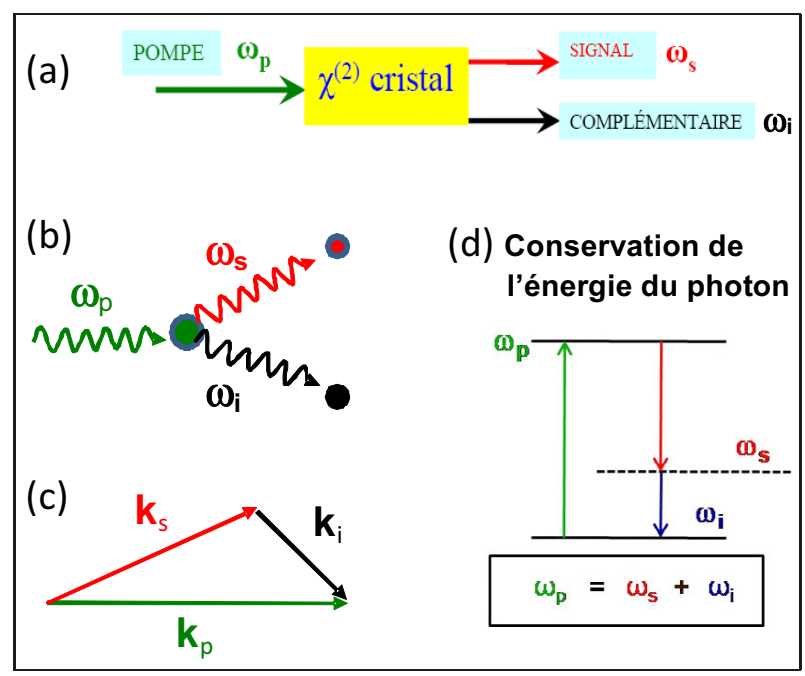

Fig. 1. - (a) et (b) : Fluorescence paramétrique (un photon pompe disparait pour donner naissance à un photon signal et un photon idler; (c) conservation de l'impulsion (condition d'accord de phase : $\Delta \mathbf{k}=\mathbf{k}_{\mathrm{p}}-\mathbf{k}_{\mathrm{s}}-\mathbf{k}_{\mathrm{i}}=0$ ) ; hors accord de phase $(\Delta \mathbf{k} \neq 0)$, la probabilité de création de la paire est proportionnelle à $\operatorname{sinc}^{2}(|\Delta \mathbf{k}| L / 2)$; (d) conservation de l'énergie du photon pompe.

(les trois ondes de polarisation identique appartenant à la même nappe d'indices). L'inversion périodique des domaines de polarisation dans un réseau (grating) de période $\Lambda=2 l_{\mathrm{c}}$ où $l_{\mathrm{c}}=\pi / \Delta \mathbf{k}$ est la longueur de cohérence de l'interaction à trois ondes (fig. 2) permet en fait de compenser le désaccord de phase $\Delta \mathbf{k} \neq 0$ par un vecteur de réseau $\mathbf{G}=2 \pi / \Lambda[10]$, de sorte que :

$$
\Delta k_{\mathrm{QPM}}=k_{\mathrm{p}}-k_{\mathrm{s}}-k_{\mathrm{i}}-\frac{2 \pi}{\Lambda}=0
$$

Il en résulte que par un choix approprié de $\Lambda$, on peut accorder en phase n'importe quel mélange paramétrique à trois ondes, d'où l'avantage des matériaux QPM par rapport aux matériaux BPM où l'existence d'un accord de phase est assujettie à la dispersion du matériau. De plus, l'accord en longueur d'onde des ondes signal et idler se fait soit par variation de la longueur d'onde du laser de pompe, soit par variation de la température ou par changement de réseau. Un dernier avantage, et non des moindres, de l'accord de phase QPM est de pouvoir coupler l'élément polaire le plus élevé $d_{33} \mathrm{du}$ tenseur de susceptibilité $\chi^{(2)}$ lorsque la polarisation des trois ondes est alignée suivant l'axe optique $c$ (confondu avec l'axe diélectrique principal $Z$ du matériau ferroélectrique, fig. 2) [8-10]. Cette découverte du quasiphasematching a révolutionné l'optique non linéaire vers le milieu des années 1990 et permis le développement de nombreuses sources OPO dans des domaines spectraux non couverts par les lasers conventionnels, notamment dans le moyen infrarouge $(2 \mu \mathrm{m}-20 \mu \mathrm{m})$.

Pour réaliser un oscillateur paramétrique, la fluorescence paramétrique très faible (le rendement de conversion d'un photon pompe est de l'ordre de $10^{-5}$ à $10^{-10}$ dû à la faiblesse de l'interaction $\chi^{(2)}$ ) doit être amplifiée de façon cohérente en plaçant le milieu non linéaire dans un

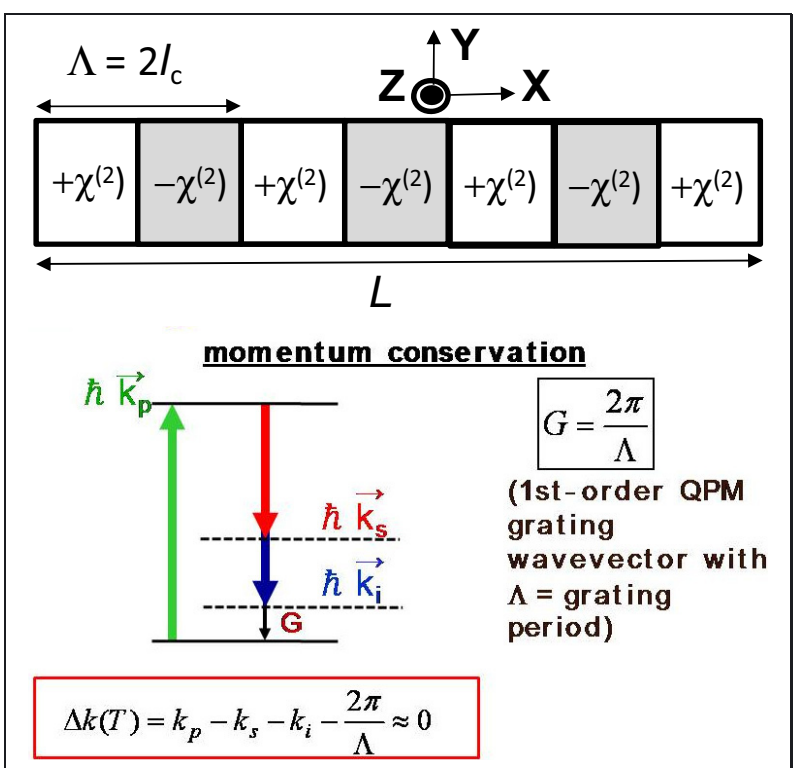

Fig. 2. - Illustration du principe du quasi-accord de phase (QPM) pour réaliser un accord de phase «à la carte». Le changement périodique du signe de la non-linéarité $\chi^{(2)}$ à chaque longueur de cohérence $l_{\mathrm{c}}$ permet de garder constant le sens du flux d'énergie (de l'onde pompe vers les ondes signal et idler). Sans retournement périodique des domaines de polarisation non linéaire, le sens du flux d'énergie s'inverse à chaque longueur de cohérence $l_{\mathrm{c}}$ et le processus paramétrique serait très faible quelle que soit la longueur du cristal. L'inversion périodique du signe de $\chi^{(2)}$ se traduit par l'apparition d'un vecteur de réseau $\mathbf{G}$ compensant le désaccord de phase dû à la dispersion normale $\Delta \mathbf{k}_{\text {norm }}=k_{\mathrm{p}}-\mathbf{k}_{\mathrm{s}}-\mathbf{k}_{\mathrm{i}} \neq 0$.

résonateur optique de très grande finesse pour l'une ou les deux ondes signal et idler. Comme pour tout oscillateur, le seuil d'oscillation est atteint lorsque le gain paramétrique égale les pertes passives sur un aller-retour dans la cavité. Plusieurs configurations de cavité sont possibles. Elles se distinguent par le nombre d'ondes résonantes simultanément dans le résonateur optique. La configuration doublement résonnante (DRO), où les deux ondes paramétriquement générées résonnent dans la cavité OPO, a un faible seuil d'oscillation (typiquement de l'ordre de $10 \mathrm{~mW}$ à $100 \mathrm{~mW}$ ), mais souffre d'instabilités de fonctionnement liées aux sauts de modes induits par la contrainte de double-résonance des ondes signal et idler dans un même résonateur $[2,4]$. En revanche la configuration simplement résonnante (SRO) (fig. 3), où l'une seule des ondes paramétriquement générées résonne, opère de manière stable mais présente un seuil d'oscillation élevé (typiquement quelques watts [5]) et nécessite donc un laser pompe puissant, disponible depuis une dizaine d'années par l'apparition de lasers fibres de puissance (jusqu'à $100 \mathrm{~W}$ en régime continu).

Le gain paramétrique en puissance $G$ (sans dimension) dans le cas où seul le signal résonne est donné par $(\operatorname{sinc}(x)=\sin (x) / x)[11,12]$

$$
\begin{aligned}
& G=\Gamma^{2} L^{2} \operatorname{sinc}^{2}(\Delta k L / 2) \\
& \Gamma^{2} L^{2}=\frac{2 \omega_{\mathrm{s}} \omega_{\mathrm{i}}|d|^{2}}{\varepsilon_{0} c^{3} n_{\mathrm{p}} n_{\mathrm{s}} n_{\mathrm{i}}} \frac{P_{\mathrm{p}}}{A} L^{2}
\end{aligned}
$$




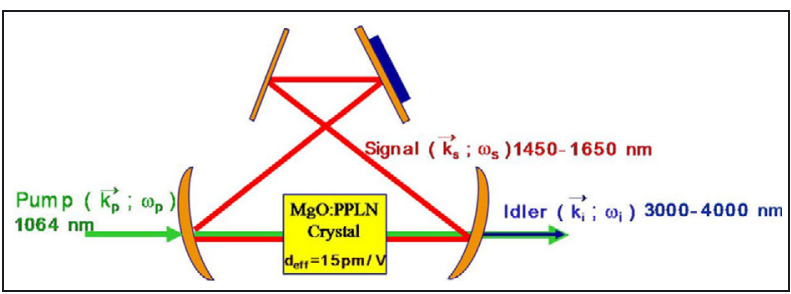

Fig. 3. - Configuration de cavité simplement résonante (SRO); seule l'onde signal est amplifiée par un traitement diélectrique approprié des miroirs de cavité.

où :

$L \quad$ est la longueur du cristal ;

$n_{\mathrm{p}, \mathrm{s}, \mathrm{i}}: n$ est l'indice de réfraction;

$d \quad$ est le coefficient non linéaire effectif $\chi_{\mathrm{eff}}^{(2)} / 2(d=$ $2 d_{33} / \pi$ dans le cas d'un accord de phase QPM d'ordre $1[10])$;

$P_{\mathrm{p}} \quad$ est la puissance incidente de pompe;

$A \quad$ est l'aire transverse moyen des faisceaux interagissant dans le cristal $\left(A \approx \pi w^{2}\right.$, où $w$ est le col du faisceau).

L'évaluation du gain pour l'OPO réalisé $\left(\lambda_{\mathrm{p}}=\right.$ $795 \mathrm{~nm}, \lambda_{\mathrm{s}}=1100 \mathrm{~nm}, \lambda_{\mathrm{i}}=2867 \mathrm{~nm}, n \approx 2,2$, $P_{\mathrm{p}} / A=3,18 \mathrm{~kW} / \mathrm{cm}^{2}$ pour $P_{\mathrm{p}}=1 \mathrm{~W}$ et $\left.w=100 \mu \mathrm{m}\right)$ donne $G=0,0158(1,6 \%)$. Comparé au gain d'un laser conventionnel basé sur une inversion de population, ce gain est 2 à 3 ordres de grandeur plus faible. Ceci explique pourquoi les pertes fractionnelles intra-cavité par aller-retour dans une cavité OPO ne peuvent pas excéder $1 \%$ à $2 \%$. Dans notre cas, le laser pompe ne peut délivrer qu'une puissance maximale de $760 \mathrm{~mW}$ à $795 \mathrm{~nm}$ (insuffisante pour un SRO), d'où la nécessité de choisir une configuration PRSRO (Pump Resonant Singly Resonant Oscillator) où l'onde pompe résonne en plus de l'onde signal dans la cavité OPO afin de permettre l'obtention d'une puissance pompe intracavité de l'ordre de $2 \mathrm{~W}$ (puissance seuil typique d'oscillation d'un SRO).

\section{Quasi-accord de phase dans le niobate de lithium périodiquement polarisé (ppMgCLN)}

Le cristal de $\mathrm{LiNbO}_{3}$ congruent à inversion de domaines de polarisation utilisé dans ce travail, est dopé par $5 \%$ d'oxyde de magnésium $(\mathrm{MgO})$ afin d'abaisser le seuil de dommage photoréfractif ( $\mathrm{ppMgCLN}$, dimension $\left.(12 \times 0,5 \times 50) \mathrm{mm}^{3}\right)$. Il a été fabriqué par HC Photonics Corp. (Taïwan) à partir d'un masque photolithographique commercial contenant neuf réseaux (A-I avec des pas $\Lambda_{0}=20,6 \mu \mathrm{m}-23,4 \mu \mathrm{m}$ ). La condition de QPM (voir Eq. (1)) peut également se mettre sous la forme suivante :

$$
\frac{n_{\mathrm{e}}\left(\lambda_{\mathrm{p}}, T\right)}{\lambda_{\mathrm{p}}}-\frac{n_{\mathrm{e}}\left(\lambda_{\mathrm{s}}, T\right)}{\lambda_{\mathrm{s}}}-\frac{n_{\mathrm{e}}\left(\lambda_{\mathrm{i}}, T\right)}{\lambda_{\mathrm{i}}}-\frac{1}{\Lambda(T)}=0,
$$

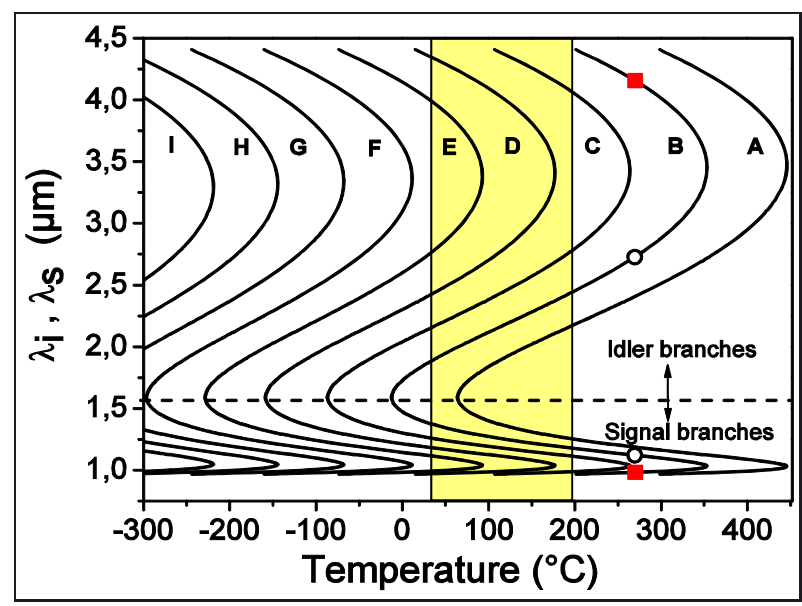

Fig. 4. - Courbes théoriques de quasi-accord de phase avec une pompe $\lambda_{\mathrm{p}}=795 \mathrm{~nm}$ pour les neuf réseaux du cristal PPLN. Les pas des réseaux $\Lambda_{0}$ spécifiés par le fabriquant sont : A : $\Lambda_{0}=20,6 \mu \mathrm{m}, \mathrm{B}: \Lambda_{0}=20,95 \mu \mathrm{m}, \mathrm{C}: \Lambda_{0}=21,3 \mu \mathrm{m}$, $\mathrm{D}: \Lambda_{0}=21,65 \mu \mathrm{m}, \mathrm{E}: \Lambda_{0}=22 \mu \mathrm{m}, \mathrm{F}: \Lambda_{0}=22,35 \mu \mathrm{m}$, $\mathrm{G}: \Lambda_{0}=22,7 \mu \mathrm{m}, \mathrm{H}: \Lambda_{0}=23,05 \mu \mathrm{m}, \mathrm{I}: \Lambda_{0}=23,4 \mu \mathrm{m}$. La zone ombrée représente la gamme de température accessible par le four utilisé, et la ligne en pointillée représente la séparation entre les branches signal et idler.

où $\lambda_{\mathrm{p}}, \lambda_{\mathrm{s}}$ et $\lambda_{\mathrm{i}}$ sont respectivement les longueurs d'onde pompe, signal et idler, vérifiant la relation de conservation de l'énergie $\lambda_{\mathrm{p}}^{-1}=\lambda_{\mathrm{s}}^{-1}+\lambda_{\mathrm{i}}^{-1}$, et $n_{\mathrm{e}}$ l'indice extraordinaire (pour une onde polarisée suivant l'axe polaire $Z$, la direction de propagation des ondes se faisant suivant l'axe diélectrique principal $X$ ). La dépendance en longueur d'onde et en température des indices $n_{\mathrm{e}}$ est donnée par les équations de Sellmeier du $\mathrm{MgO}: \mathrm{LiNbO}_{3}$ [13]. Notons que la condition d'accord de phase (2) doit tenir compte de la dilatation thermique du pas des réseaux QPM, $\Lambda(T)=\Lambda_{0} f\left(T-T_{0}\right)$, où $\Lambda_{0}$ est la période à $T_{0}=22{ }^{\circ} \mathrm{C}$ [14]. Les courbes de quasi-accord de phase théorique réseau par réseau (calculées à partir de la relation (3)) sont représentées en fonction de la température $\mathrm{du}$ cristal sur la figure 4 .

La figure 4 montre que seulement cinq réseaux (A-E) satisfont à la condition de QPM pour une pompe à $795 \mathrm{~nm}$ (correspondant au pic de gain du laser Ti:saphir) et une gamme de température entre $30{ }^{\circ} \mathrm{C}$ et $200{ }^{\circ} \mathrm{C}$. Du fait de la dispersion du $\mathrm{LiNbO}_{3}$ et de la courte longueur d'onde pompe $\left(\lambda_{\mathrm{p}}=795 \mathrm{~nm}\right)$, il existe deux paires $\left(\lambda_{\mathrm{s}}, \lambda_{\mathrm{i}}\right)$ vérifiant la condition de QPM à chaque température (chaque paire étant matérialisée soit par deux carrés, soit par deux cercles). Cependant seule une des deux paires pourra osciller à cause du processus de compétition de modes longitudinaux dans un laser à élargissement homogène du gain, comme c'est le cas d'un OPO. Parmi les deux solutions possibles (correspondant aux deux branches sur la fig. 4), celle qui expérimentera le gain le plus fort (ou les pertes les plus faibles) oscillera. Nous verrons plus loin que c'est la branche signal supérieure $\left(\lambda_{\mathrm{s}}>1,1 \mu \mathrm{m}\right)$ correspondant à la branche idler inférieure $\left(\lambda_{\mathrm{i}}<3,5 \mu \mathrm{m}\right)$ qui oscillera, limitant ainsi les longueurs d'onde pouvant être générées dans 


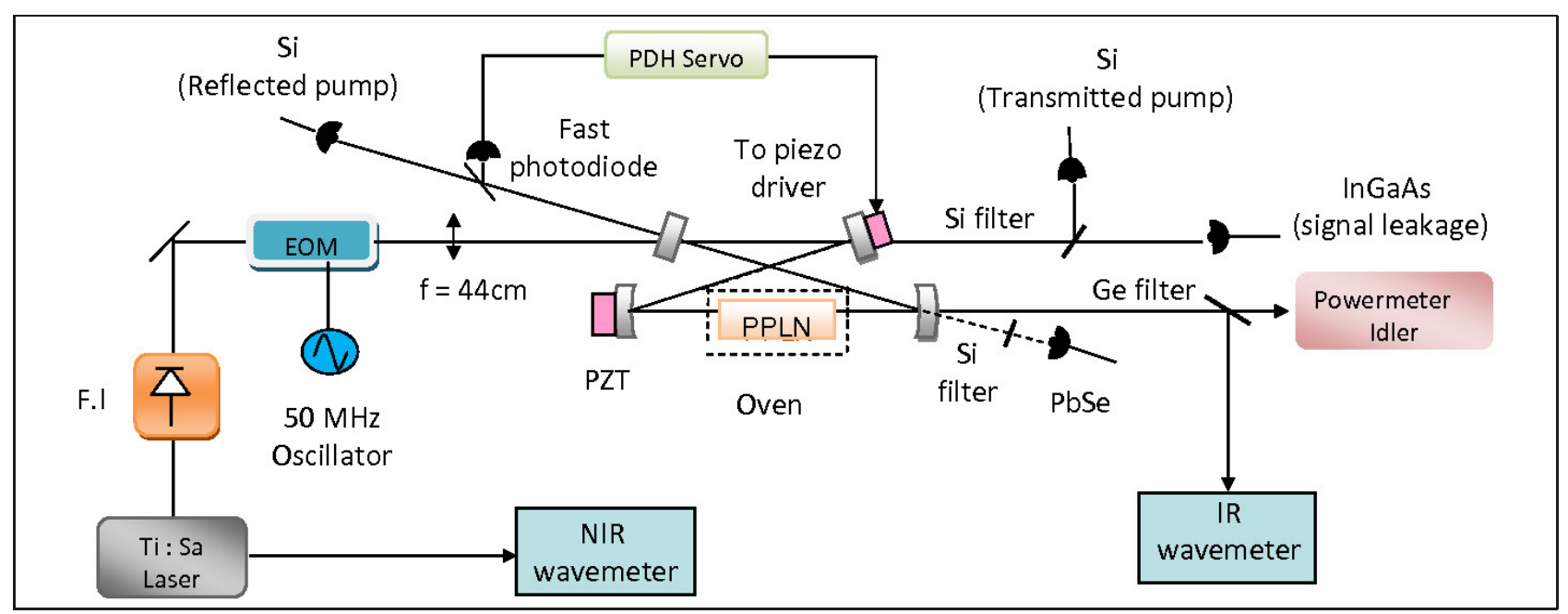

Fig. 5. - Dispositif expérimental du PRSRO; la cavité OPO (centre de la figure) est une cavité en anneau formée de deux miroirs sphériques et de deux miroirs plans. L'onde pompe est couplée dans la cavité par un coupleur plan $\left(T_{\mathrm{p}}=5 \%\right)$, après avoir été modulée en phase à $f_{\mathrm{m}}=50 \mathrm{MHz}$ par un modulateur électro-optique (EOM) pour créer les deux bandes latérales $\left(\omega_{\mathrm{p}} \pm 50 \mathrm{MHz}\right)$ utilisées en réflexion pour asservir la cavité sur la fréquence porteuse $\omega_{\mathrm{p}}$. Le cristal de ppMgCLN (PPLN) est monté dans un four régulé en température. L'onde idler ressort de la cavité par le miroir sphérique de droite. Un filtre en Germanium sous incidence de Brewster (transmettant au-delà de $1,8 \mu \mathrm{m}$ ) sert à couper le résidu de pompe et de signal colinéaire au faisceau idler.

l'IR à 3,5 $\mu \mathrm{m}$. Pour des longueurs d'onde pompe plus grandes $\left(\lambda_{\mathrm{p}}>850 \mathrm{~nm}\right)$, les courbes de QPM ne présentent plus qu'une seule branche signal (branche inférieure) et idler (branche supérieure) [15] : pour pouvoir générer $\lambda_{\mathrm{i}}>3,5 \mu \mathrm{m}$, il faudrait donc pomper l'OPO à $\lambda_{\mathrm{p}}>850 \mathrm{~nm}$, ce qui est impossible car la puissance du laser Ti:saphir chute considérablement à ces longueurs d'onde, sauf à changer les miroirs de cavité.

\section{Dispositif expérimental et asservissement}

Le dispositif expérimental est illustré par la figure 5. Le laser de pompe est un laser Ti:saphir en anneau continu monomode et accordable (Matisse TR de Spectra Physics). Il est utilisé à $795 \mathrm{~nm}$ où la puissance disponible est de $760 \mathrm{~mW}$ lorsqu'il est pompé avec un laser $\mathrm{Nd}: \mathrm{YVO}_{4}$ doublé en fréquence de $5 \mathrm{~W}$ (Millenia de Spectra-Physics). Sa largeur de raie est inférieure à $2 \mathrm{MHz}$, et $\lambda_{\mathrm{p}}$ peut être balayée continûment (sans saut de mode) sur $\sim 50 \mathrm{GHz}$. Pour asservir la cavité sur la longueur d'onde pompe, on utilise la technique de PoundDrever-Hall (PDH) $[16,17]$. La fréquence de pompe est modulée en phase par un modulateur électro-optique (EOM) résonnant à $f_{\mathrm{m}}=50 \mathrm{MHz}$, dans le but de transférer $5 \%$ de la porteuse dans les bandes latérales.

La puissance pompe maximale disponible en entrée de la cavité OPO chute à $490 \mathrm{~mW}$ à cause des pertes introduites par l'isolateur optique (rapport d'isolation $-40 \mathrm{~dB}$ ) et les différents éléments optiques se trouvant sur le trajet du faisceau pompe. La cavité OPO est une cavité en anneau constituée par deux miroirs plans (M1 et M2) et deux miroirs sphériques (M3 et M4) de rayon de courbure égale $r=-100 \mathrm{~mm}$. La longueur totale de la cavité est de $485 \mathrm{~mm}$ avec une distance M3-M4 valant $135 \mathrm{~mm}$ (ainsi l'intervalle spectral libre est égal à FSR $=550 \mathrm{MHz}$ en tenant compte d'un indice de réfraction de 2,21 pour
$\mathrm{LiNbO}_{3}$ ) ce qui nous donne un waist $w_{\mathrm{s}}$ (col de faisceau) égal à $60 \mu \mathrm{m}$ (à $\lambda_{\mathrm{s}}=1,4 \mu \mathrm{m}$ ) au centre du cristal PPLN. Le demi-angle de pliage de la cavité OPO est pris égal à $11^{\circ}$ (valeur minimale imposée par les dimensions du four dans lequel est logé le cristal PPLN) dans le but de limiter les effets d'astigmatisme des miroirs sphériques utilisés hors d'axe. De plus la cavité OPO a été conçue de telle façon à ce que le paramètre de focalisation $\xi=L / 2 z_{\mathrm{p}}\left(z=(1 / 2) k w^{2}\right.$ est la longueur de Rayleigh, $k=2 \pi n / \lambda)[18]$ satisfasse $\xi \geq 1(\xi=1,5)$ pour maximiser le gain paramétrique, tout en limitant les pertes par diffraction sur les faces d'entrée-sortie du cristal PPLN (épaisseur du cristal de $500 \mu \mathrm{m}$, longueur $L=50 \mathrm{~mm}$,). Comme les ondes pompe et signal résonnent dans la même cavité, ils ont une longueur de Rayleigh identique $\left(z_{\mathrm{p}}=z_{\mathrm{s}}\right)$ ce qui implique un waist signal de $w_{\mathrm{s}}=105 \mu \mathrm{m}$ sur les faces du cristal suite à la propagation du centre (où il vaut $w_{\mathrm{s}}=60 \mu \mathrm{m}$ ) vers la face de sortie, une valeur 4 fois plus petite l'épaisseur du cristal afin de limiter les pertes par diffraction sur les faces d'entrée-sortie du cristal.

Le coupleur d'entrée de la cavité OPO a un revêtement dichrö̈que qui permet une transmission partielle pour les longueurs d'onde comprise entre $790 \mathrm{~nm}$ et $810 \mathrm{~nm}\left(\operatorname{avec} T_{\mathrm{p}}=5 \%\right.$ à $\left.\lambda_{\mathrm{p}}=795 \mathrm{~nm}\right)$ et une haute réflectivité pour les ondes signal $\left(R_{\mathrm{s}}=99,9 \%\right.$ pour $\left.0,97 \mathrm{~nm}<\lambda_{\mathrm{s}}<1,6 \mu \mathrm{m}\right)$. Les autres miroirs de la cavité OPO sont hautement réfléchissant aux longueurs d'onde pompe et signal $\left(R_{\mathrm{p}, \mathrm{s}}>99,9 \%\right)$ et hautement transmissifs pour les longueurs d'onde idler $\left(T_{\mathrm{i}}>75 \%\right.$ pour 1,7 $\left.\mu \mathrm{m}<\lambda_{\mathrm{i}}<4,5 \mu \mathrm{m}\right)$. De plus le cristal ppMgCLN est traité antireflet sur la bande du signal $\left(R_{\mathrm{S}}<0,5 \%\right.$ par face) et sur la pompe $\left(R_{\mathrm{p}}<0,6 \%\right.$ par face $)$ afin de limiter les pertes passives dans la cavité et abaisser ainsi le seuil d'oscillation. En revanche le cristal transmet plus de $95 \%$ de l'idler généré. Le cristal est logé dans un four dont la température est régulée au dixième de degré 


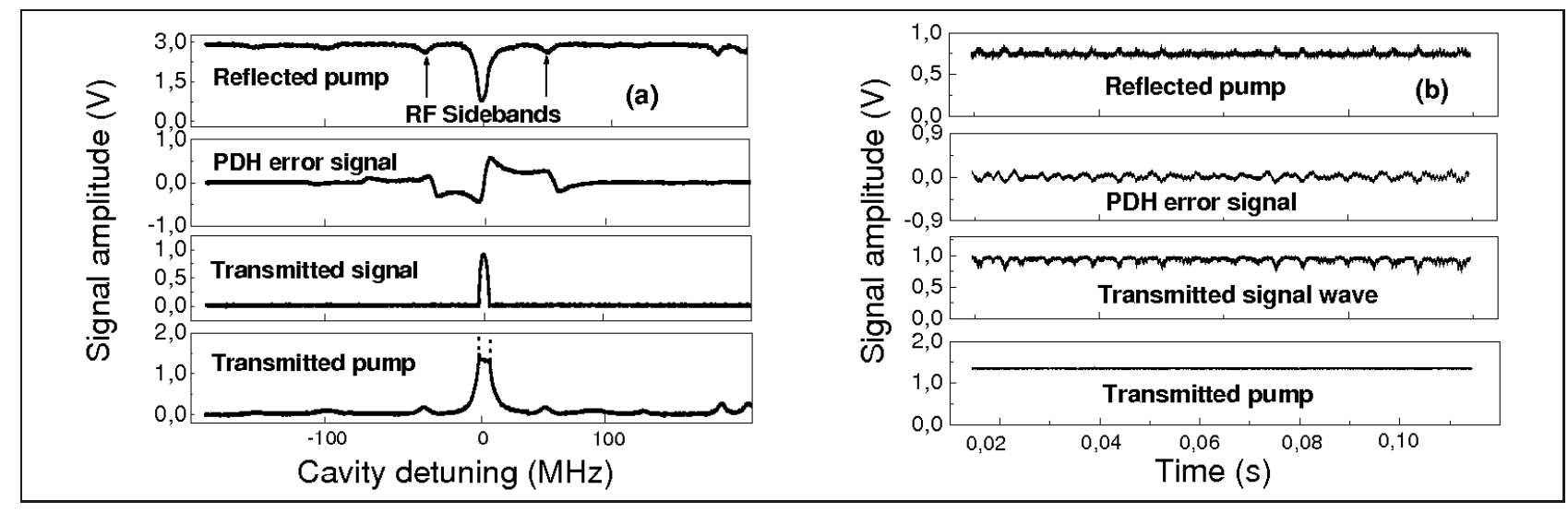

Fig. 6. - de bas en haut : (i) frange de pompe en transmission montrant l'effet de clamping au seuil en fonction du balayage de la longueur de cavité sur $\Delta \mathrm{L}_{\text {cav }}=0,2 \mu \mathrm{m}$; (ii) frange signal transmise; (iii) signal d'erreur dispersif PDH permettant d'asservir la longueur de la cavité OPO sur la porteuse $\omega_{\mathrm{p}}$ (i.e. au minimum de la frange en réflection (iv)); (b) Mêmes signaux en fonction du temps lorsque la boucle de verrouillage est actionnée.

$\left(\Delta T= \pm 0,1^{\circ} \mathrm{C}\right)$, l'ensemble étant monté sur un système mécanique permettant d'ajuster les degrés de liberté suivant $\theta, \varphi, X, Y$. Cette stabilité en température du four est suffisante car l'acceptance en température du quasiaccord de phase est de $\Delta T=3{ }^{\circ} \mathrm{C}$.

Afin de coupler le maximum de puissance de pompe dans la cavité PRSRO, le mode transverse TEM $_{00}$ du faisceau pompe doit correspondre au waist de pompe propre de la cavité $\left(w_{\mathrm{p}}=260 \mu \mathrm{m}\right)$ entre les deux miroirs plans M1-M2 $\left(w_{\mathrm{p}}=\left(\lambda_{\mathrm{p}} / \lambda_{\mathrm{s}}\right)^{1 / 2} w_{\mathrm{s}}=45 \mu \mathrm{m}\right.$ au centre du cristal car $\left.z_{\mathrm{p}}=z_{\mathrm{s}}\right)$. L'adaptation de mode du faisceau pompe à la cavité $\mathrm{OPO}$ est réalisée par une lentille de focale $f=450 \mathrm{~mm}$.

L'efficacité d'adaptation de mode obtenue (mode matching) est supérieure à $95 \%$ car le mode transverse du faisceau laser est TEM $_{00}$. L'efficacité de l'adaptation d'impédance (impedance matching [19]) est estimée à partir du contraste des franges pompe en réflexion (fig. 6). Elle s'améliore de $70 \%$ en dessous du seuil d'oscillation à $80 \%$ au-dessus du seuil d'oscillation grâce aux pertes non linéaires additionnelles liées à la conversion paramétrique. La puissance pompe stockée dans la cavité est estimée à partir du faisceau pompe résiduel transmis par le miroir plan M2 solidaire de la céramique PZT (fig. 5) et détecté par une photodiode au silicium calibrée (en V/W). L'onde signal résiduelle transmise par le filtre silicium est détecté par une photodiode InGaAs pour détecter les franges signal (fig. 6).

Une des difficultés liée à la configuration PRSRO (par rapport à SRO) est l'obligation de maintenir la permanence de la résonance de pompe dans la cavité OPO. Sans asservissement, il est impossible de maintenir la condition de résonance à cause des bruits acoustiques perturbant (sur une échelle sub-micrométrique) la longueur de la cavité en anneau. Pour maintenir en résonance la fréquence $\omega_{\mathrm{p}}$ sur une frange d'Airy de la cavité bruitée, on utilise le signal d'erreur dispersif (i.e. impair et passant pas zéro) issu du battement entre la porteuse $\omega_{\mathrm{p}}$ et ses deux bandes latérales réfléchies (fig. 5). Ce battement à

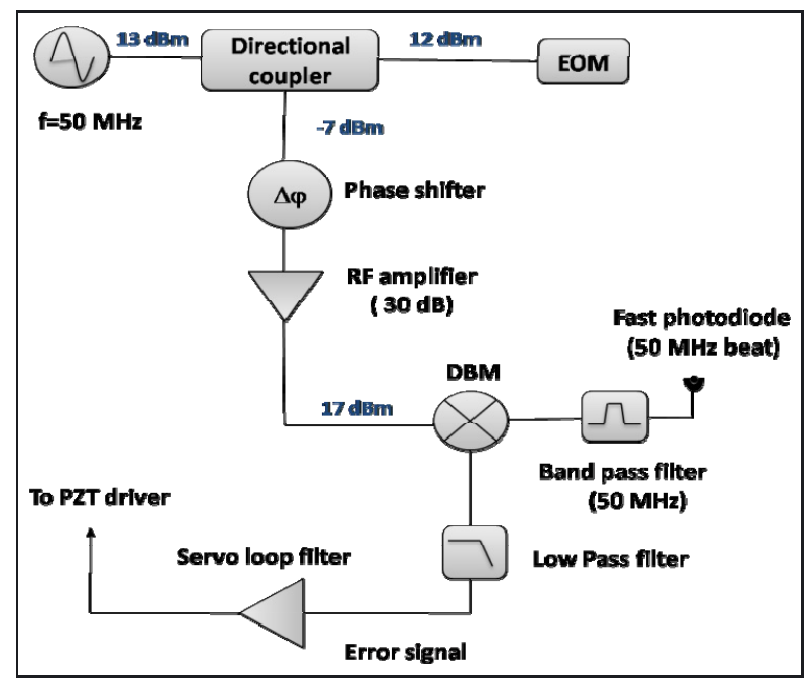

Fig. 7. - Schéma électronique d'élaboration et de démodulation du signal d'erreur PDH. Le signal électrique de battement détecté par la photodiode Si est d'abord filtré autour de $f_{\mathrm{m}}$ puis mélangé avec la référence $f_{\mathrm{m}}$ préalablement déphasé et amplifié dans le mélangeur équilibré (DBM), avant d'être démodulé par un filtre passe-bas $\left(f_{\mathrm{c}}<1 \mathrm{MHz}\right)$ et intégré par un filtre correcteur pour fournir le signal de correction, lequel est envoyé à travers un amplificateur HV à la céramique PZT.

$f_{\mathrm{m}}=50 \mathrm{MHz}$ (qui contient l'information sur les fluctuations acoustiques de la cavité encodées au voisinage de $50 \mathrm{MHz}$ ) qui s'annule lorsque la porteuse est exactement en résonance avec la frange d'Airy (désaccord de cavité $\delta_{\mathrm{p}}=v_{\mathrm{p}}-v_{\mathrm{cav}}=0$ ) est détecté par une photodiode $\mathrm{Si}$ rapide et ensuite démodulé vers les basses fréquences de Fourier pour donner la courbe (iii) de la figure 6a lorsque la longueur de cavité est balayée autour de la frange d'Airy.

La figure 7 présente le schéma électronique de la démodulation du signal d'erreur. L'avantage de la technique d'asservissement $\mathrm{PDH}$ en mode réflexion par rapport à d'autres méthodes couramment utilisées en asservissement laser tient à sa grande plage de capture 


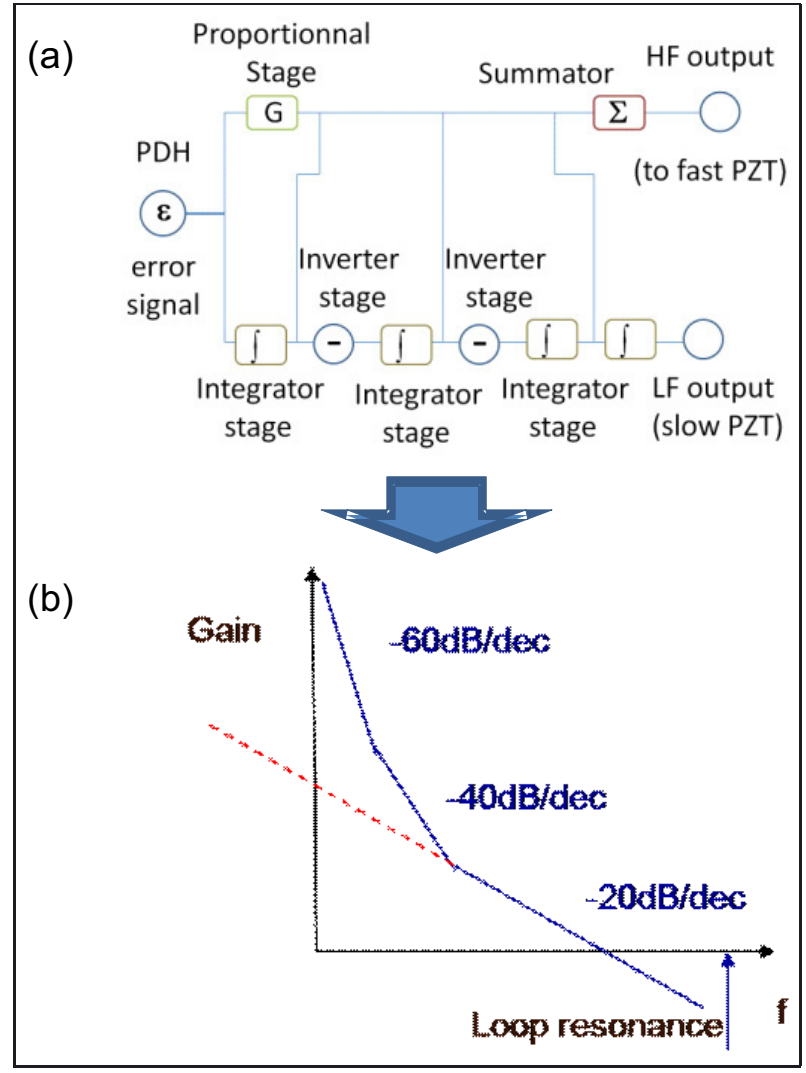

Fig. 8. - (a) Schéma électronique de principe du filtre correcteur; le signal de correction est séparé en deux voies (proportionnelle $\mathrm{P}$ et intégrale I). La voie intégrale comprend un $1^{\mathrm{er}}$ intégrateur pur dont la sortie est sommée avec la voie proportionnelle. Un $2^{\mathrm{e}}$ intégrateur réintègre la sortie du premier (après une inversion de signe pour des raisons de marge de phase) et sa sortie également sommée à la voie $\mathrm{P}$. Un troisième intégrateur est rajoutée en cascade. La sortie du sommateur est envoyée sur une PZT rapide pilotant l'un des miroirs plan (fig. 5). La sortie du $3^{\mathrm{e}}$ intégrateur contenant les corrections lentes est finalement ré-intégrée et envoyée sur la $2^{\mathrm{e}}$ céramique PZT afin de préserver la dynamique de l'ampli HT de la voie rapide ; (b) Fonction de transfert du filtre correcteur. Les trois intégrateurs en cascade permettent d'affecter un gain élevé pour les fréquences de Fourier basses $(<200 \mathrm{~Hz})$ tout en évitant d'exciter la première résonance de la PZT rapide (flèche) qui se trouve vers $2 \mathrm{kHz}$.

$\left( \pm f_{\mathrm{m}}\right)$ en cas de perturbation acoustique de grande amplitude $[16,17]$, conférant une certaine robustesse à l'asservissement. D'autre part cette technique (utilisée en mode réflexion) permet en principe de corriger des fluctuations de phase très rapides d'un laser de pompe (bien que dans notre cas où la correction est appliquée à une cavité de surtension, la bande passante d'asservissement est limitée par les résonances piézo-électriques de la PZT à quelques kilohertz). En effet, les bandes latérales n'étant pas transmises par la cavité mais directement réfléchies sans subir le délai de transit dans celle-ci (à l'inverse de la porteuse), le signal d'erreur contient par conséquent l'information sur ces fluctuations rapides.

Le schéma du filtre correcteur Proportionnel-Intégral (P-I) utilisé est représenté sur la figure $8 \mathrm{a}$ ainsi que le gain en boucle ouverte du filtre (fig. 8b). Le filtre utilise une

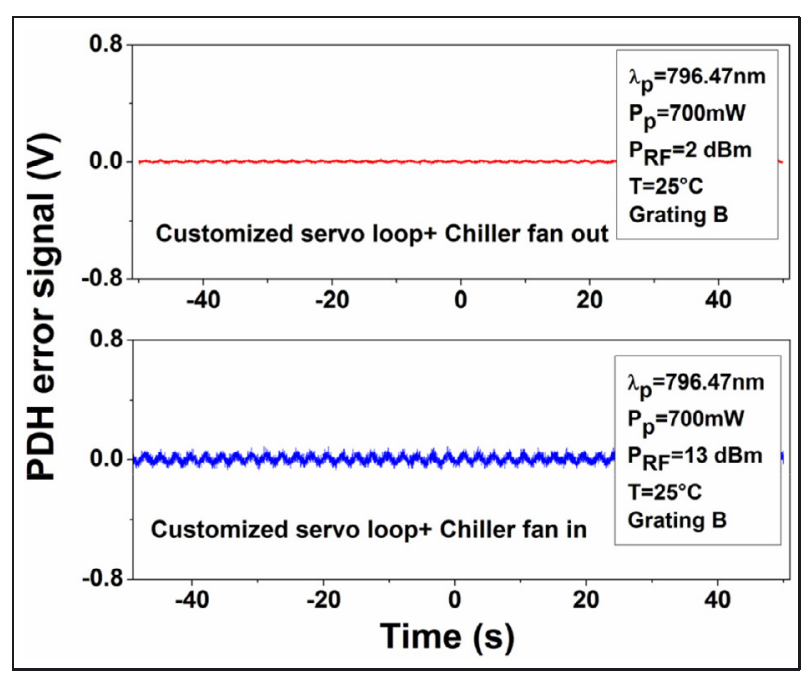

Fig. 9. - Signal d'erreur PDH obtenu avec le filtre correcteur de la figure 8 ; en bas : en présence du bruit généré par le chiller du cristal de Ti:saphir; en haut : chiller déplacé hors de la salle.

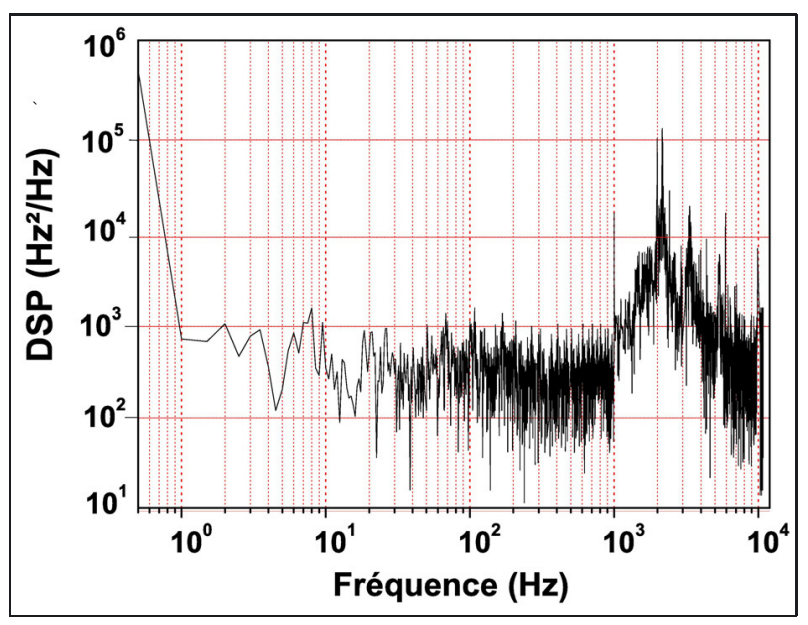

Fig. 10. - Densité spectrale de bruit de fréquence résiduelle de l'asservissement PDH.

cascade de trois intégrateurs pour la partie I afin de corriger parfaitement les fluctuations acoustiques basse fréquence, tout en évitant d'exciter la première résonance piézo-électrique des céramique PZT (vers $2 \mathrm{kHz}$ ). La figure 9 illustre les performances du filtre correcteur à trois intégrateurs en cascade sur le signal d'erreur PDH.

Le panneau inférieur a été enregistré en présence du système de refroidissement à eau (chiller) bruyant du barreau de Ti:saphir du laser de pompe. Lorsque le chiller est évacué dans la pièce adjacente, l'effet d'atténuation du bruit acoustique basse fréquence du chiller est clairement mis en évidence. Comparée à la trace (iii) de la figure $6 \mathrm{~b}$, obtenue avec un système d'asservissement commercial P-I comportant un simple intégrateur de gain $-20 \mathrm{~dB} /$ decade, une nette amélioration des performances de l'asservissement est constatée.

La figure 10 montre la densité spectrale de bruit de fréquence résiduel $S_{v-\text { cav }}(f)$ du signal d'erreur PDH en boucle fermée ( $f$ est la fréquence de Fourier), calculée d'après le signal temporel de la figure 9 (haut). Cette 
courbe renseigne sur les fluctuations résiduelles de la frange d'Airy de la cavité par rapport à la fréquence de pompe $v_{\mathrm{p}}$. Nous voyons sur le spectre que la bande passante BW de l'asservissement est d'environ $2 \mathrm{kHz}$, limitée par la première résonance piézo-électrique de la céramique PZT.

En boucle fermée, cet asservissement maintient la cavité PRSRO verrouillée sur la fréquence du laser de pompe pendant plus de $30 \mathrm{~min}$. On peut alors balayer la fréquence $v_{p}$ sur environ $1,5 \mathrm{GHz}$ (la cavité suit sans se déverrouiller), ce qui est la plage de balayage typique sans saut de mode d'un PRSRO [20]. Au-delà, l'asservissement décroche car un saut de mode signal intervient alors suite au changement de longueur de la cavité : ceci est une conséquence de la contrainte de double-résonance de la pompe et du signal dans une même cavité. Une solution pour augmenter la plage d'accord sans saut de mode de l'onde idler d'un PRSRO consiste à faire résonner séparément la pompe et le signal dans une cavité duale à deux bras, de façon à contrôler indépendamment la longueur des deux sous-cavités [20,21]. Dans le cas d'un OPO simplement résonant sur le signal (SRO) et accordé par balayage en fréquence de la pompe, la situation est différente de celle d'un PRSRO : la longueur de cavité restant inchangée pendant que la fréquence de pompe varie, la fréquence de l'onde signal qui oscille ne change pas pendant le balayage [22]. Ceci permet alors, même sans asservissement de la cavité SRO, de transférer l'intégralité de l'excursion pompe sur l'idler, pouvant alors résulter en un balayage sans saut de mode de l'idler sur environ $150 \mathrm{GHz}$.

Une fois le PRSRO asservi (à $v_{\mathrm{p}}$ fixée), la puissance moyen-IR (idler) présente toutefois une dérive décroissante lente sur plusieurs dizaines de minutes. Dans les applications nécessitant un niveau de puissance IR stable, une stabilisation active de la puissance idler au moyen (par exemple d'un modulateur acousto-optique) s'avèrera nécessaire.

\section{Analyse des performances du PRSRO}

\subsection{Signature dynamique de l'oscillation dans un PRSRO : effet de limitation de puissance pompe}

La figure 6a montre les puissances pompe et signal transmises et réfléchie par la cavité OPO ainsi que le signal d'erreur lorsque la cavité est balayée au voisinage d'une frange d'Airy. Une signature de l'oscillation paramétrique dans le cas d'un PRSRO se traduit par le clampage de la puissance pompe transmise à sa valeur seuil (fig. 6a(i)) [23]. Ce comportement de limitation de puissance de la pompe intra-cavité à sa valeur seuil, révélé théoriquement par Siegman dès 1961 [24], est caractéristique des OPOs résonant sur la pompe comme également dans le cas d'un OPO triplement résonant (TRO) [25,26]. Ce phénomène de clampage peut être rapproché de la dynamique d'un laser conventionnel à élargissement de gain homogène : dès que le seuil d'oscillation est atteint (i.e. gain $=$ pertes sur un aller-retour de cavité) le gain dû à l'inversion de population cesse de croître et sature à sa valeur seuil, signifiant que tout apport d'énergie supplémentaire dû à la pompe est convertie en énergie laser (dans la cas d'un PRSRO en puissance signal-idler). Cependant la résonance de l'onde pompe dans un PRSRO limite l'efficacité de la conversion paramétrique à l'inverse d'un OPO simplement résonant sur l'onde signal (SRO), à cause du phénomène d'adaptation d'impédance (impedance matching) selon lequel la puissance pompe stockée dans un résonateur optique n'est optimale que lorsque les pertes fractionnelles $\varepsilon_{\mathrm{L}}$ sur un aller-retour dans le résonateur égalent le coefficient de transmission $T_{\mathrm{p}} \mathrm{du}$ coupleur d'entrée, soit $T_{\mathrm{p}}=\varepsilon_{\mathrm{L}}$. Le résonateur n'étant pas passif en présence de conversion non linéaire, les pertes fractionnelles sont donc non linéaires dans un PRSRO et valent $\varepsilon_{\mathrm{L}}+\varepsilon_{\mathrm{NL}}\left(P_{\text {in }}\right)$ où $P_{\text {in }}$ est la puissance laser incidente. Il s'ensuit alors que l'adaptation d'impédance non linéaire n'est parfaitement réalisée que pour une puissance de pompe injectée donnée $P_{\text {in }}$ choisie comme étant la puissance maximale $P_{\max }$ disponible à l'entrée du résonateur. Ainsi lorsque $P_{\text {in }}>P_{\max }$, la puissance additionnelle $P_{\text {in }}-P_{\text {max }}$ sera réfléchie par le résonateur. Nous aborderons cette notion d'impedance matching optimale dans la partie 5.5, à savoir comment choisir $T_{\mathrm{p}}$ pour une puissance donnée $P_{\max }$. Expérimentalement l'observation des franges de pompe en réflexion (fig. 6a(iv)) permet d'observer ce phénomène d'impedance matching non linéaire lorsque la puissance pompe injectée est augmentée à partir du seuil d'oscillation : le contraste des franges de pompe en réflexion (fig. 6a(iv)) augmente alors de $70 \%$ à $80 \%$ (i.e. la puissance de pompe réfléchie par la cavité pour cause d'inadaptation d'impédance diminue).

La figure $6 \mathrm{~b}$ représente l'évolution temporelle des puissances pompe (i) et signal (ii) transmises, de la puissance pompe réfléchie (iv) ainsi que du signal d'erreur PDH (iii) en boucle fermée (de valeur moyenne nulle), lorsque la cavité OPO est asservie sur la longueur d'onde pompe. Rappelons que cette figure correspond à un asservissement non optimal de la cavité sur le laser de pompe (utilisation d'un lockbox commercial). Une remarque frappante est la quasi-absence du bruit observé sur les autres signaux sur la trace du signal pompe intracavité clampé, d'où la notion de power limiting introduite par Siegman relative à la pompe transmise par ce dispositif non linéaire [24].

\subsection{Seuil d'oscillation dans un PRSRO}

Cette puissance seuil intra-cavité est indépendante de la puissance pompe incidente, et est donnée par la relation (4) [23] :

$$
P_{\mathrm{p}}^{\text {cav }}(\text { seuil })=\frac{T_{\mathrm{s}}+V_{\mathrm{s}}}{E}
$$

Dans l'équation (4), $T_{\mathrm{s}} \ll 1$ est le coefficient de transmission en puissance du coupleur de sortie M4 relatif à l'onde signal et $V_{\mathrm{s}}$ les pertes passives subies par l'onde signal sur un tour de cavité $(1 \%)$. Le coefficient de non linéarité $E$ prend en compte le caractère gaussien des faisceaux pompe, signal et idler ainsi 


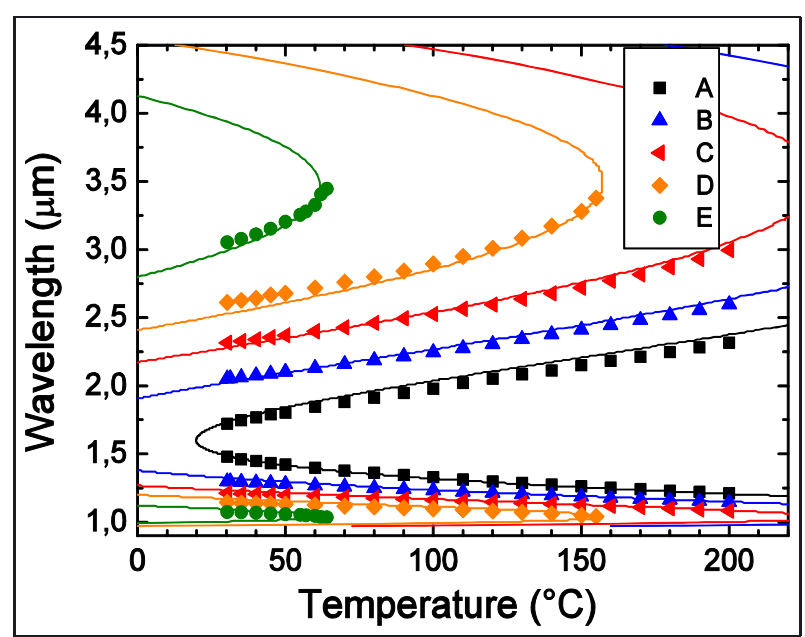

Fig. 11. - Courbes expérimentales de quasi-accord de phase (réseaux A-E : $\Lambda_{0}=20,8 \mu \mathrm{m}-22,13 \mu \mathrm{m}$ à $T=22{ }^{\circ} \mathrm{C}$, $\lambda_{\mathrm{p}}=795 \mathrm{~nm}$ ). Le point de dégénérescence de l'OPO (à $\lambda_{\mathrm{s}}=$ $\lambda_{\mathrm{i}}=\lambda_{\mathrm{p}} / 2=1,59 \mu \mathrm{m}$ ) sépare les deux branches signal des deux branches idler. Les courbes en trait plein sont calculées à partir de l'équation (3) en utilisant les relations de dispersion du MgO:LiNbO ${ }_{3}$ mais en changeant de $\sim 1 \%$ la période des réseaux par rapport aux valeurs spécifiées par le constructeur (fig. 4). L'OPO préfère osciller sur la branche signal supérieure (branche idler inférieure, $\lambda_{\mathrm{i}} \leq 3,5 \mu \mathrm{m}$ ) pour laquelle la paire $\left(\lambda_{\mathrm{s}}, \lambda_{\mathrm{i}}\right)$ possède un seuil moindre à cause de l'absorption du matériau au-delà de $\lambda=3,8 \mu \mathrm{m}$.

que le coefficient non linéaire du PPLN. Il a pour expression $E=4 \mu_{0} d_{\mathrm{eff}}^{2} \omega_{\mathrm{s}}^{2} \omega_{\mathrm{i}}^{2} L h(\xi) /\left(\pi c^{2} n_{\mathrm{p}}^{2} \omega_{\mathrm{p}}^{2}\right)\left(\right.$ en $\left.\mathrm{W}^{-1}\right)$ où $d_{\text {eff }}=2 d_{33} / \pi$ est le coefficient non linéaire du ppMgCLN, et la fonction $h(\xi)$ est la fonction de focalisation qui vaut $h=1$ pour un seuil minimum obtenue pour un waist de pompe optimum $[18,23]$. Pour un cristal de longueur $L=50 \mathrm{~mm}$, un coefficient non linéaire effectif $d_{\mathrm{eff}}=14 \mathrm{pm} / \mathrm{V}$, un waist $w_{\mathrm{p}} \sim 50 \mu \mathrm{m}$ et $T_{\mathrm{p}}=0,05$ à $795 \mathrm{~nm}$, le seuil pompe intra-cavité expérimental peut être estimé à partir de la frange pompe en transmission de la figure $6 \mathrm{a}$, et vaut 1,3 W. Cette valeur de puissance seuil est en accord avec le seuil d'oscillation d'un SRO pour la même longueur de cristal PPLN [5]. Par ailleurs il est intéressant de noter que la frange pompe en réflexion (fig. 6a(iv)) ne présente pas de phénomène d'écrêtement (clamping) observé sur la frange pompe en transmission (fig. 6a(i)). Ceci peut s'expliquer par le fait que très au dessus du seuil, la porteuse directement réfléchie due à l'inadaptation d'impédance pompe domine par rapport à la faible fraction du champ pompe intra-cavité qui fuit de cette dernière. Ce qui justifie du choix de l'élaboration du signal d'erreur PDH en mode réfection, car la pente relativement abrupte du signal d'erreur (fig. 6a(iii)) autour de sa valeur nulle n'est pas affectée par le clampage (ce qui n'aurait pas été le cas si la frange pompe transmise avait été utilisée pour le PDH).

\subsection{Caractérisation des courbes d'accord de phase}

La figure 11 montre la caractérisation spectrale de l'émission infrarouge des cinq réseaux utilisables du ppMgCLN en fonction de la température (à comparer avec les courbes théoriques de la fig. 4). Tel que nous l'avons prédit en partie 2, le PRSRO oscille toujours sur la branche haute du signal correspondant à la branche basse de l'idler $\left(1,59 \mu \mathrm{m}<\lambda_{\mathrm{i}}<3,5 \mu \mathrm{m}\right)$ du fait d'une absorption plus importante du cristal PPLN aux longueurs d'onde idler supérieures à $3,8 \mu \mathrm{m}$ proche de la zone de coupure totale $\left(\lambda_{\text {cutoff }} \approx 4,5 \mu \mathrm{m}\right)$ de la transparence du $\mathrm{LiNbO}_{3}$. Les pertes par absorption de l'onde idler (même si celle-ci n'oscille pas) influent aussi sur le seuil d'oscillation de l'OPO, car elles sont vues comme des pertes indirectes sur la pompe responsables d'un seuil d'oscillation plus élevée.

De plus on remarque que les courbes de QPM expérimentales sont translatées de $-40{ }^{\circ} \mathrm{C}$ vers les températures basses par rapport aux courbes théoriques de la figure 4 . Cet écart important est lié aux incertitudes (inférieures au pourcent) sur la périodicité des pas de réseau du cristal telles que spécifiées par le fabricant (l'écart n'est en aucun cas dû à la fiabilité des équations de Sellmeier utilisées [13]). Ce décalage des courbes expérimentales de QPM par rapport aux courbes théoriques ainsi que l'oscillation du PRSRO sur les branches idler du bas a également été rapporté par Lindsey et al., qui ont utilisé une pompe à $810 \mathrm{~nm}[20,27]$.

Néanmoins le choix d'une longueur d'onde pompe plus grande (vers $850 \mathrm{~nm}$ ) permet l'oscillation du PRSRO sur la branche idler du haut (entre $4 \mu \mathrm{m}$ et $5 \mu \mathrm{m}$ ) [28].

Du fait de la translation des courbes de quasi-accord de phase expérimentales vers les faibles températures, le point de dégénérescence $\left(\lambda_{\mathrm{s}, \mathrm{i}}=\lambda_{\mathrm{p}} / 2\right)$ du réseau $\mathrm{A}$ se retrouve à $T=21{ }^{\circ} \mathrm{C}$ (au lieu de $60{ }^{\circ} \mathrm{C}$ sur la fig. 4). $\mathrm{Au}$ voisinage de la température ambiante, le signal et l'idler sont proches de la dégénérescence $\left(\lambda_{\mathrm{s}}=1590 \mathrm{~nm}\right.$, $\lambda_{\mathrm{i}}=1620 \mathrm{~nm}$ ) et tous deux tombent dans la bande HR des miroirs de cavité $(1,1 \mu \mathrm{m}-1,7 \mu \mathrm{m})$ : l'oscillation paramétrique subit alors une transition de la configuration PRSRO vers la configuration triplement résonante (TRO) de seuil plus bas. Nous avons pu constater qu'entre $21^{\circ} \mathrm{C}$ et $35^{\circ} \mathrm{C}$, le fonctionnement du PRSRO sur ce réseau était en effet instable vis-à-vis des sauts de mode (fig. 12) [4]. De plus au voisinage de ce point de dégénérescence, les fluctuations de puissance signal sont très importantes, et il devient impossible d'asservir la cavité OPO en dessous de $T=35^{\circ} \mathrm{C}$ à cause de ces instabilités de modes.

\subsection{Puissance idler générée $(1,8 \mu \mathrm{m}-3,5 \mu \mathrm{m})$}

La figure 13 montre la puissance idler en fonction de la puissance pompe incidente à $T=140{ }^{\circ} \mathrm{C}$ et pour les différents pas de réseaux correspondants à différentes longueurs d'onde idler (Eq. (3)). Les puissances idler maximales sont obtenues avec le réseau $\mathrm{A}$, et correspondent aux longueurs d'onde idler les plus courtes (l'efficacité du processus paramétrique étant proportionnelle à $\left.\lambda_{\mathrm{p}} /\left(\lambda_{\mathrm{i}}-\lambda_{\mathrm{p}}\right)\right)$. La courbe en trait plein correspond à un ajustement par la méthode des moindres carrés des points expérimentaux à la formule (5) [23] :

$$
P_{\mathrm{i}}^{\text {out }}=4 \frac{\omega_{\mathrm{i}}}{\omega_{\mathrm{s}}} \frac{T_{\mathrm{p}}}{T_{\mathrm{p}}+V_{\mathrm{p}}} P_{\mathrm{th}}^{\text {in }}\left(\sqrt{\frac{P_{\mathrm{in}}}{P_{\text {in }}^{\text {th }}}}-1\right),
$$




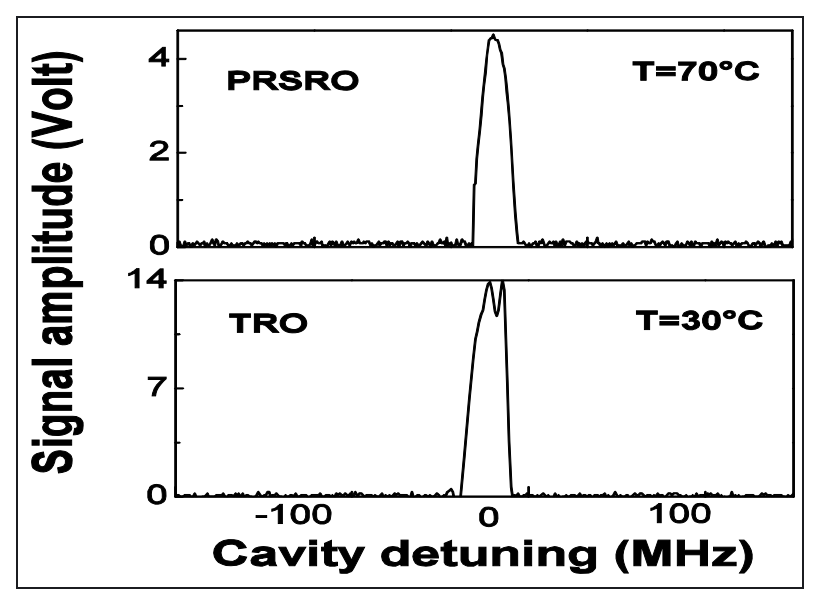

Fig. 12. - Frange signal générée par le réseau A lorsque la cavité est balayée au voisinage d'une résonance de pompe à $T=70{ }^{\circ} \mathrm{C}$ (comportement d'un PRSRO) et à $T=30^{\circ} \mathrm{C}$ (comportement d'un TRO). On peut voir sur la frange TRO un double pic correspondant à un saut de paires de mode signal-idler pendant le court laps de temps où la pompe passe en résonance.

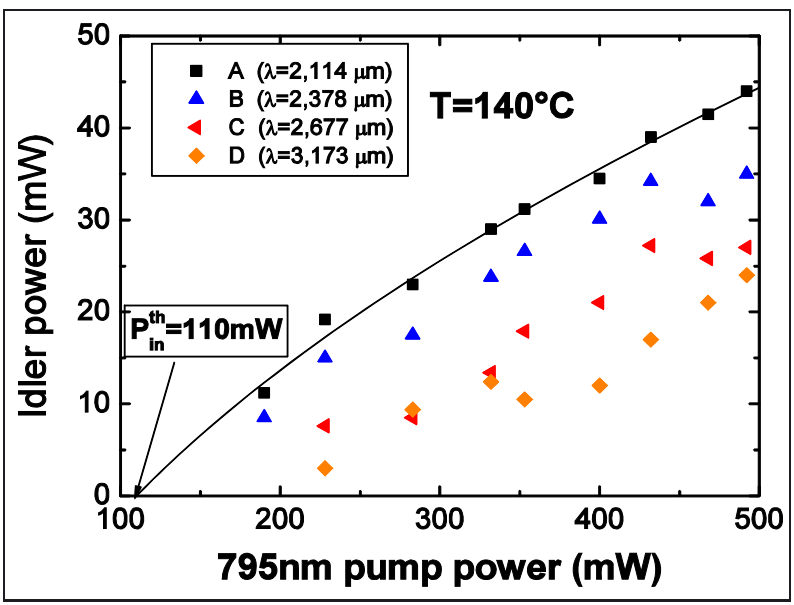

Fig. 13. - Puissance idler en fonction de la puissance pompe pour les cinq réseaux du chip à une température donnée. La courbe en trait continu est un ajustement par les moindres carrés à la formule (3), donnant un seuil de $110 \mathrm{~mW}$. L'efficacité décroissante lorsque $\lambda_{\mathrm{i}}$ augmente est intrinsèque au processus de down-conversion (voir fig. 14) : plus l'idler se situe dans l'IR plus l'efficacité du processus non linéaire décroit.

où $T_{\mathrm{p}}$ est le coefficient de transmission pompe du coupleur d'entrée $\left(T_{\mathrm{p}}=5 \%\right)$ et $V_{\mathrm{p}}$ les pertes passives fractionnelles vues par l'onde pompe sur un tour de cavité (excluant $T_{\mathrm{p}}$ ). $P_{\text {in }}$ est la puissance pompe réellement couplée dans la cavité OPO (compte tenu des adaptations de mode et d'impédance imparfaites) et $P_{\text {in }}^{\text {th }}$ la puissance seuil extra-cavité déduite de la puissance seuil intra-cavité donnée par l'équation (4) :

$$
P_{\text {in }}^{\text {th }}=\frac{T_{\mathrm{p}}+V_{\mathrm{p}}}{T_{\mathrm{p}}} \frac{\left(T_{\mathrm{p}}+V_{\mathrm{p}}\right)\left(T_{\mathrm{s}}+V_{\mathrm{s}}\right)}{4 E}
$$

L'expression de la puissance seuil extra-cavité est obtenue en divisant l'équation (4) par le facteur de qualité de la cavité à longueur d'onde pompe.

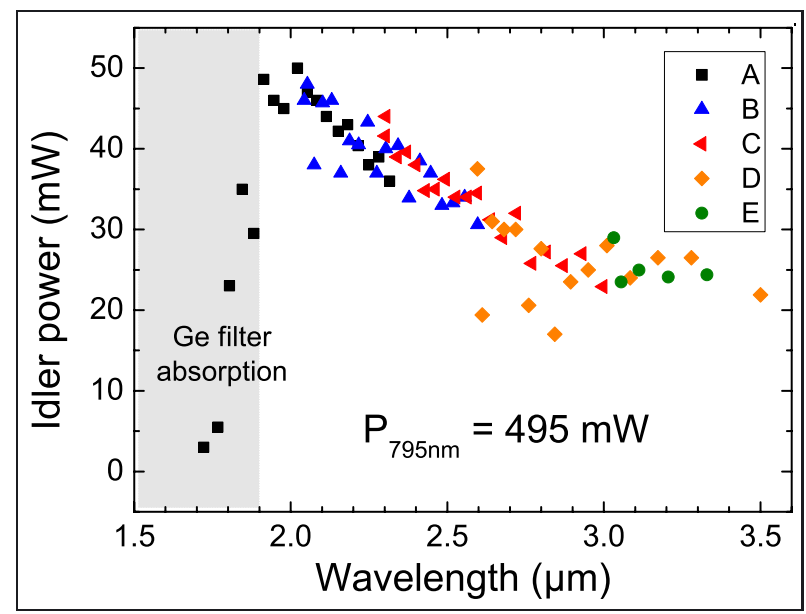

Fig. 14. - Puissance idler (tous réseaux confondus) en fonction de la longueur d'onde; le filtre en Ge (utilisé pour isoler les photons idler des photons pompe et signal résiduels) atténue par absorption la puissance réelle idler détectée en dessous de $\lambda_{i}=$ $2 \mu \mathrm{m}$; la courbe en trait plein est ajustée d'après l'équation (5) qui prédit une dépendance en $\omega_{\mathrm{i}} / \omega_{\mathrm{s}}=\lambda_{\mathrm{p}} /\left(\lambda_{\mathrm{i}}-\lambda_{\mathrm{p}}\right)$.

L'ajustement des points expérimentaux obtenus à partir du réseau A donne une puissance seuil $\left(P_{\mathrm{i}}=0\right)$ externe égale à $110 \mathrm{~mW}$, illustrant l'effet de la résonance de pompe. Cette valeur est équivalente à celle obtenue par Turnbull et al. [20] avec une longueur d'onde pompe et de cristal similaires aux nôtres. Compte tenu de la valeur estimée du seuil intracavité au paragraphe 5.2, le facteur de surtension $Q_{p}$ de la cavité est de 13 pour le coupleur d'entrée utilisé $\left(T_{\mathrm{p}}=0,05\right)$. Nous verrons plus loin que cette valeur de $T_{\mathrm{p}}$ ne correspond probablement pas à une adaptation d'impédance optimale, compte tenu des pertes de conversion non linéaires dans la cavité.

La figure 14 montre la couverture spectrale IR sur une octave de longueur d'onde du PRSRO $\left(1,8 \mu \mathrm{m}<\lambda_{\mathrm{i}}<\right.$ $3,5 \mu \mathrm{m})$ tous réseaux confondus utilisés entre $T=30{ }^{\circ} \mathrm{C}$ et $T=200^{\circ} \mathrm{C}$. La décroissance de l'efficacité de conversion paramétrique avec la longueur d'onde idler est liée à la dépendance en $\omega_{\mathrm{i}} / \omega_{\mathrm{s}}=\lambda_{\mathrm{p}} /\left(\lambda_{\mathrm{i}}-\lambda_{\mathrm{p}}\right)$ de la puissance idler qui est intrinsèque au phénomène de conversion paramétrique. La puissance maximum IR $(50 \mathrm{~mW})$ est générée autour de $\lambda_{\mathrm{i}}=2 \mu \mathrm{m}$.

Il est à noter qu'en dessous de $2 \mu \mathrm{m}$, cette puissance mesurée est en fait atténuée par le filtre en germanium (Ge) dont la longueur d'onde de coupure est $\lambda_{\mathrm{c}}=1,8 \mu \mathrm{m}$. L'utilisation d'un filtre adéquat nous permettrait d'avoir des puissances idler de l'ordre de $60 \mathrm{~mW}$ à $\lambda_{\mathrm{i}}=1,8 \mu \mathrm{m}$. Ces puissances sont environ 2 à 3 ordres de grandeurs plus élevées que pour une source laser DFG (obtenue par différence de fréquences de deux lasers pompe), illustrant l'avantage d'un oscillateur OPO par rapport à un processus simple de DFG, même exhalté en cavité [7]. En fait les rendements de conversion mesurés sont les plus élevés rapportés dans les publications. Pour couvrir la branche supérieure de l'idler $\left(3,5 \mu \mathrm{m}<\lambda_{\mathrm{i}}<4,2 \mu \mathrm{m}\right)$, il faudra pomper le PRSRO à $\lambda_{\mathrm{p}} \geq 850 \mathrm{~nm}$, ce qui nécessite de changer le jeu des miroirs du laser Ti:saphir 
afin d'optimiser sa puissance à ces longueurs d'onde et d'atteindre le seuil d'oscillation.

\subsection{Adaptation d'impédance optimale dans un PRSRO}

La question de l'adaptation d'impédance dans un $\mathrm{OPO}$ où l'onde pompe résonne n'a jamais été adressée dans la littérature. Dans une cavité passive, l'adaptation d'impédance optimale est réalisée lorsque le coefficient de transmission pompe $T_{\mathrm{p}}$ égale la somme de toutes les pertes passives sur un aller-retour, excluant $T_{\mathrm{p}}$ lui-même. Dans un résonateur non linéaire comme le PRSRO, il faut tenir compte des pertes non linéaires dues à la conversion paramétrique [19]. Ces pertes non linéaires dépendant de la puissance pompe, l'optimisation de $T_{\mathrm{p}}$ doit forcément être corrélée avec la puissance de pompe $P_{\text {in }}$ à l'entrée de l'OPO.

Nous avons revisité la théorie du PRSRO [23] afin de prédire, pour une puissance maximale $P_{\text {in }}$ à l'entrée de la cavité, quelle est le coefficient de transmission optimal $T_{\mathrm{p}}^{\mathrm{opt}}$ qui réalise un impedance matching parfait, qui se traduit par un couplage total dans la cavité (i.e. pour lequel la puissance de pompe réfléchie par la cavité est nulle). En supposant $T_{\mathrm{s}, \mathrm{p}} \ll 1$, on peut démontrer qu'il existe une valeur optimale de $T_{\mathrm{p}}$ qui réalise une adaptation d'impédance non linéaire optimale [29],

$$
T_{\mathrm{p}}^{\mathrm{opt}}=\eta_{\mathrm{NL}} P_{\mathrm{in}} \frac{1-T_{\mathrm{s}} / 2}{2 T_{\mathrm{s}}},
$$

où $\eta_{\mathrm{NL}}=\left[4 \omega_{\mathrm{s}} \omega_{\mathrm{i}} d^{2} /\left(\varepsilon_{0} c^{3} n_{\mathrm{p}} n_{\mathrm{s}} n_{\mathrm{i}}\right)\right]\left(L^{2} / \pi w_{\mathrm{p}}^{2}\right)$ (exprimé en $\left.\mathrm{W}^{-1}\right)$. Nous voyons ainsi que plus la non linéarité du cristal $d$ est élevée, plus un coupleur transmissif est adéquat. Il en va de même pour la puissance maximale $P_{\text {in }}$. Le fait que $T_{\mathrm{p}}^{\mathrm{opt}}$ dépende de la puissance pompe illustre la notion d'adaptation d'impédance non linéaire, contrairement au cas d'une cavité ne contenant que des pertes passives. Pour construire un PRSRO, il convient par conséquent de connaître préalablement la puissance de pompe maximale disponible, afin de prédire la valeur du coefficient adéquat du coupleur d'entrée.

La figure 15 montre l'illustration théorique de l'existence d'un tel optimum pour $T_{\mathrm{p}}$, où $\Gamma_{\mathrm{i}}=N_{\mathrm{i}} / N_{\mathrm{p}}^{\text {in }}$ est le ratio du nombre de photons idler généré par rapport au nombre de photons pompe incidents sur la cavité et $\Gamma_{\mathrm{p}}=N_{\mathrm{p}}^{\mathrm{refl}} / N_{\mathrm{p}}^{\text {in }}$ le ratio du nombre de photons pompe réfléchis par rapport au nombre de photons pompe incidents. On constate alors qu'à l'optimum $T_{\mathrm{p}}^{\mathrm{opt}}(0,05$ dans le cas de la figure) tous les photons pompe sont convertis en paires signal-idler $\left(\Gamma_{\mathrm{p}}=0, \Gamma_{\mathrm{i}}=100 \%\right)$.

Nous avons pu vérifier expérimentalement l'existence d'un tel optimum pour $T_{\mathrm{p}}$, réalisant une adaptation d'impédance optimale pour laquelle quasiment toute la puissance de pompe injectée dans la cavité est convertie par le processus paramétrique. Comme nous ne disposions pas de coupleur d'entrée à variable à $\lambda_{\mathrm{p}}=795 \mathrm{~nm}$, nous avons profité des oscillations du coefficient de réflexion du coupleur au voisinage de $800 \mathrm{~nm}\left(5 \%<T_{\mathrm{p}}<11 \%\right.$ entre $\lambda_{\mathrm{p}}=790 \mathrm{~nm}$ et $810 \mathrm{~nm}$, symboles carrés noirs sur

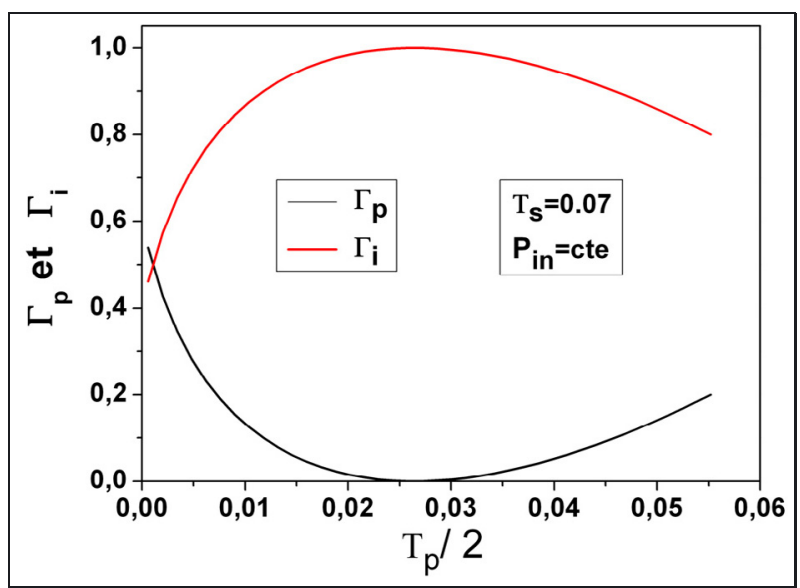

Fig. 15. - Efficacité quantique de conversion idler $\left(\Gamma_{\mathrm{i}}\right)$ et dépeuplement de la pompe $\left(\Gamma_{\mathrm{p}}\right)$ dans la cavité en fonction du coefficient de transmission du coupleur d'entrée, à une puissance pompe incidente $P_{\text {in }}$ fixée à plusieurs fois le seuil. Une conversion de $100 \%$ est réalisée pour $T_{\mathrm{p}}=0,05$.

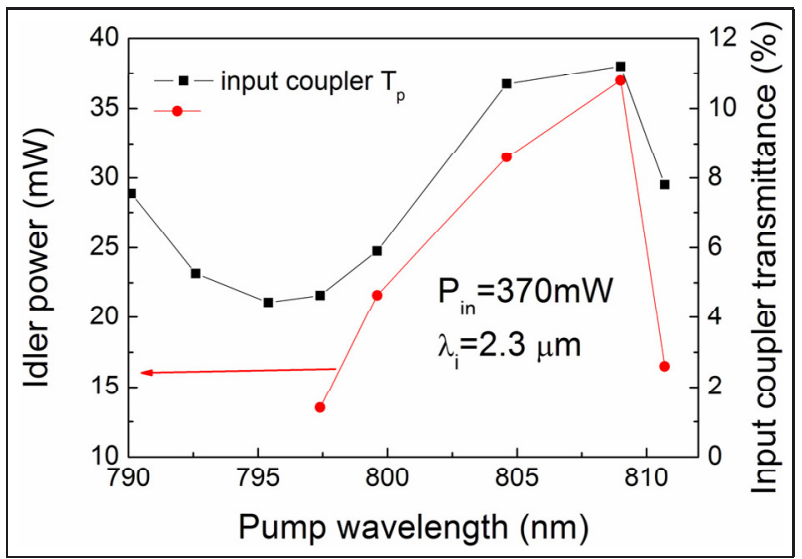

Fig. 16. - Adaptation d'impédance pompe optimale par variation du coefficient $T_{\mathrm{p}}$ à une puissance de pompe incidente fixée ; la variation de $T_{\mathrm{p}}$ utilise les rebonds du traitement diélectrique partiellement réflecteur du coupleur d'entrée au voisinage de $800 \mathrm{~nm}$.

la fig. 16) pour faire varier $T_{\mathrm{p}}$ en changeant la longueur d'onde de pompe. Ce faisant, il a fallu pour chaque point (cercles) de la figure 14 changer la température du ppMgCLN afin que $\lambda_{\mathrm{i}}$ reste constante (ici $\lambda_{\mathrm{i}}=2,3 \mu \mathrm{m}$ ). De plus, nous avons fixé la puissance incidente $P_{\text {in }}$ à $370 \mathrm{~mW}$ entre $790 \mathrm{~nm}$ et $810 \mathrm{~nm}$, un niveau de puissance garantissant que le PRSRO oscille au-dessus du seuil sur toute la plage de variation de $T_{\mathrm{p}}$. On voit sur la figure 16 qu'à pompage constant, la puissance idler (représentée en cercles pleins) est optimale à $\lambda_{\mathrm{p}}=808 \mathrm{~nm}$ malgré un seuil plus élevé (d'après (6)), correspondant à $T_{\mathrm{p}}^{\mathrm{opt}}=0,11$ (11\%). La plage de variation de $\lambda_{\mathrm{p}}$ est suffisamment petite pour que tous les autres paramètres (non linéarité du cristal, pertes passives sur les trois autres miroirs $R_{\max }$ de la cavité) puissent être considérés comme constants dans l'intervalle considéré. Bien évidemment, pour une autre valeur de la puissance incidente $P_{\text {in }}$, la valeur de $T_{\mathrm{p}}^{\mathrm{opt}}$ varie également suivant l'équation (7), qui peut ainsi être considérée comme une équation utile pour optimiser la 
puissance de sortie d'un PRSRO une fois la puissance de pompe maximale disponible connue.

\section{Conclusions et perspectives}

Nous avons réalisé un oscillateur paramétrique optique pompé par un laser titane-saphir continu résonant à la fois sur la pompe et le signal (PRSRO), et délivrant une puissance supérieure à $20 \mathrm{~mW}$ sur une octave de longueur d'onde IR entre $1,7 \mu \mathrm{m}$ et 3,5 $\mu \mathrm{m}$. Cette puissance est largement suffisante pour les applications radiométriques envisagées. Plusieurs améliorations des performances du PRSRO restent à être apportées, notamment sur la couverture spectrale au-delà de $3,5 \mu \mathrm{m}$. La puissance idler devra être asservie en intensité avant de pouvoir l'envoyer sur le radiomètre cryogénique du laboratoire. Pour l'extension vers $4 \mu \mathrm{m}-4,5 \mu \mathrm{m}$, il sera nécessaire de changer les miroirs du laser titane-saphir pour en optimiser l'émission vers $\lambda_{\mathrm{p}}=850 \mathrm{~nm}-860 \mathrm{~nm}$.

Le maximum de gain du laser se situant plutôt vers $795 \mathrm{~nm}$, une puissance de pompe disponible moindre (sans doute autour de $300 \mathrm{~mW}-350 \mathrm{~mW}$ ) est attendue, résultant en un pompage proche du seuil et donc des instabilités de fonctionnement. Pour y remédier, on peut envisager de remplacer le Millenia-5W par un laser de pompe vert $(532 \mathrm{~nm})$ fournissant $10 \mathrm{~W}$. La puissance du laser Ti:saphir à $795 \mathrm{~nm}$ sera alors doublée, de l'ordre de 1,3 W, au lieu de $700 \mathrm{~mW}$ actuellement. Bien qu' une telle puissance ne permette pas d'envisager l'utilisation d'une configuration de cavité SRO plus stable (ne nécessitant pas une exaltation de la puissance pompe $[5,22])$, elle permettra cependant de pomper le PRSRO à $850 \mathrm{~nm}$ afin de couvrir le domaine 3,5 $\mu \mathrm{m}-4,5 \mu \mathrm{m}$ sans avoir besoin de changer les optiques du laser Ti:saphir.

La construction de cet OPO constitue une première étape réalisant l'extension vers l'infrarouge moyen des sources cohérentes disponibles au laboratoire non seulement pour les applications métrologiques en radiométrie et photométrie, mais également pour la spectroscopie infrarouge d'espèces moléculaires à l'état de traces (détection de polluants atmosphériques, analyse spectroscopie du souffle humain, détections de résidus chimiques de combustion de processus industriels, détection de traces d'explosifs ou d'espèces chimiques nocifs, etc.). À moyen terme, nous envisagerons l'extension de la chaîne radiométrique vers le bleu $(\sim 390$ nm-450 nm) par doublement de fréquence du laser Ti:saphir dans une cavité de résonance externe. Avec cet OPO il est également possible de couvrir le domaine spectral vert-rouge (500 nm-700 nm) par doublage intracavité (du PRSRO) de l'onde signal fortement résonant, en plaçant le cristal doubleur sur le deuxième waist de la cavité (entre les deux miroirs M1 et M2 sur la fig. 2b). Pour ce faire, une modification de la cavité OPO s'avèrera nécessaire en remplaçant ces deux miroirs plans par deux miroirs sphériques de rayon de courbure adéquat de façon à créer un waist secondaire suffisamment petit entre les miroirs plans M1 et M2, et donc optimiser l'efficacité intracavité du processus non linéaire de doublement de fréquence (SHG).
À plus long terme, une fois l'extension vers le bleu réalisée, nous envisagerons l'extension vers l'ultraviolet par triplement de fréquence indirect du titane-saphir $(\omega+2 \omega \rightarrow 3 \omega)$, par mélange non linéaire du bleu généré avec l'émission fondamentale. Il est cependant clair que la source laser primaire commerciale (laser Ti:saphir), déjà sollicitée par de nombreuses applications au laboratoire, devra être dupliquée.

Cet article constitue une version longue de notre article paru récemment dans Applied Physics B [30]. Ces recherches ont bénéficié partiellement du financement européen FP7/iMERA+ (Joint Research project T2.J02 Breath analysis for early disease diagnosis, contrat $\mathrm{N}^{\circ} 217257$ ).

\section{Références}

[1] Kosterev A., Wysocki G., BAKHIRKIN Y., So S., LEWicki R., Fraser M., TitTel F. et CURL R.F., "Application of quantum cascade lasers to trace gas analysis", Appl. Phys. B, 90, 2007, 165-176.

[2] SCHILLER S., "Optical parametric oscillators (continuous wave)", Encyclopedia of modern optics, 4, 2004, 51-61.

[3] Strossner U., MEYN J.P., WALLENSTEIN R., URENSKI P., Arie A., Rosenman G., Mlynek J., Schiller S. et Peters A., "Single-frequency continuous-wave optical parametric oscillator system with an ultrawide tuning range of 550 to $2830 \mathrm{~nm}$ ", J. Opt. Soc. Am B, 19, 2002, 1419-1424.

[4] Eckardt R.C., NABors C.D., Kozlovsky W.J. et BYER R.L., "Optical parametric oscillator frequency tuning and control", J. Opt. Soc. Am. B, 8, 1991, 646-666.

[5] YANG S.T., EcKardT R.C. et BYer R.L., "Continuouswave singly resonant optical parametric oscillator pumped by a single-frequency resonantly doubled Nd:YAG laser", Opt. Lett., 18, 1993, 971-973.

[6] Maddaloni P., Gagliardi G., Malara P. et De NATALE P., "A 3.5-mWcontinuous-wave differencefrequency source around $3 \mu \mathrm{m}$ for sub-Doppler molecular spectroscopy", Appl. Phys. B, 80, 2004, 141-145.

[7] Witinski M.F., PAUl J.B. et ANDERson J.G., "Pump enhanced difference-frequency generation at $3.3 \mu \mathrm{m}$ ", Appl. Opt., 48, 2009, 2600-2606.

[8] YARIV A., Quantum Electronics, $3^{\text {rd }}$ ed., Chap. 17 (Wiley, 1988, ISBN 0-471-60997-6).

[9] HUM D.S. et FEJER M.M., "Quasi-phasematching", C.R. Physique, 8, 2007, 180-198.

[10] Myers L.E., Eckardt R.C., FeJer M.M., Byer R.L., BOSENBERG W.R. et PIERCE J.W., "Quasi-phase-matched optical parametric oscillators in bulk periodically poled $\mathrm{LiNbO}_{3}$ ", J. Opt. Soc. Am. B, 12, 1995, 2102-2116.

[11] HARRIS S.E., "Tunable Optical Parametric Oscillators", Proc. IEEE, 57, 1969, 2096-2113.

[12] SUTHERLAND R.L., Handbook of Nonlinear Optics, $2^{\text {nd }}$ edition, Chap. 3, ed. Marcel Dekker, New York, 2003. 
[13] Gayer O., Sacks Z., Galun E. et ARIE A., "Temperature and wavelength dependent refractive index equations for $\mathrm{MgO}$-doped congruent and stoichiometric $\mathrm{LiNbO}_{3}$ ", Appl. Phys. B, 91, 2008, 343-348.

[14] Paul O., Quosig A., Bauer T., NitTmann M., BARTSVHKe J., ANSTETT G. et L'HUILliER J.A., "Temperature-dependent Sellmeier equation in the MIR for the extraordinary refractive index of $5 \% \mathrm{MgO}$ doped congruent LiNbO3", Appl. Phys. B, 86, 2007, 111-115.

[15] Stothard D.J.M., EbrahimZadeh M. et DUNN M.H., "Low-pump-threshold continuous-wave singly resonant optical parametric oscillator", Opt. Lett., 23, 1998, 18951897.

[16] DReVer R.W.P., Hall J.L., Kowalski F.V., Hough J., FORD G.M., MUNLEY A.J. et WARD H., "Laser phase and frequency stabilization using an optical resonator", Appl. Phys. B, 31, 1983, 97-105.

[17] BLACK E.D., "An introduction to Pound-Drever-Hall laser frequency stabilization", Am. J. Phys., 69, 2001, 7987.

[18] Boyd G.D. et KleInMan D.A., "Parametric interaction of focused Gaussian light beams", J. Appl. Phys., 39, 1968, 3597-3639.

[19] Note : Dans un résonateur optique, l'adaptation d'impédance consiste à coupler le maximum de puissance de l'onde incidente, compte tenu des pertes sur un aller-retour (pertes passives ou/et non linéaires comme c'est le cas pour un OPO car la conversion d'une partie de la puissance pompe vers le signal et l'idler est vue par la cavité comme étant des pertes dynamiques additionnelles proportionnelles à la puissance pompe intra-cavité). L'adaptation est optimale lorsque le coefficient de transmission $T_{\mathrm{p}}$ du coupleur d'entrée égale les pertes (linéaires et non linéaires) sur un aller-retour.

[20] Turnbull G.A., McGloin D., Lindsay I.D., EBRAHIMZADEH M. et DUNN M.H., "Extended modehop-free tuning by use of a dual-cavity, pump-enhanced optical parametric oscillator", Opt. Lett., 25, 2000, 341-343.

Article reçu le 18 mars 2011, version révisée reçue le 16 décembre 2011.
[21] MÜller F., PopP A. et KüHNEMANN F., “Transportable, highly sensitive photoacoustic spectrometer based on a continuous-wave dual-cavity optical parametric oscillator", Opt. Express, 11, 2003, 2820-2825.

[22] ANDRIEUX E., ZANON T., CAdORET M., RiHAN A. et ZONDY J.-J., "500-GHz mode-hop-free idler tuning range with a frequency-stabilized signal-resoant optical parametric oscillator", Opt. Lett., 36, 7, 2011, 1212-1214.

[23] SCHILlER S., SCHNEIDER K. et MLYNEK J., "Theory of an optical oscillator with resonant pump and signal", J. Opt. Soc. B, 16, 1999, 1512-1524.

[24] SiEgman A.E., "Nonlinear Optical Effects: An Optical Power Limiter", Appl. Opt., 1, 1962, 127-132.

[25] Lugiato L., Oldano C., Fabre C., Giacobino E. et HoROwICZ R., "Bistability, self-pulsing and chaos in optical parametric oscillators", Nuovo Cimento D, 10, 1988, 959-977.

[26] Debuisschert T., Sizmann A., Giacobino E. et FAbre C., "Type-II continuous-wave optical parametric oscillators: oscillation and frequency-tuning characteristics", $J$. Opt. Soc. Am. B, 10, 1993, 1668-1680.

[27] Lindsay I.D., Petridis C., DUNN M.H. et EBRAHIMZADEH M., "Continuous-wave pump-enhanced singly resonant optical parametric oscillator pumped by an extended-cavity diode laser", Appl. Phys. Lett., 78, 2001, 871-873.

[28] Ebrahimzadeh M., Philips P.J. et Das S., "Lowthreshold mid-infrared optical parametric oscillator in periodically-poled LiNbO3 synchroneously puped by a Ti:Sapphire laser", Appl. Phys. B, 72, 2001, 793-801.

[29] Rihan A., Zanon-Willette T., AndRIEuX E., CADORET M. et ZONDY J.-J., "Perturbative theory of continuous-wave optical parametric oscillators", soumis à Phys. Rev. A., 2011.

[30] RiHAN A., ANDRIEUX E., ZANON-Willette T., BRIAUDEAU S., HiMBERT M. et ZONDY J.-J., "A pumpresonant signal-resonant optical parametric oscillator for spectroscopic breath analysis", Appl. Phys. B, 102, 2011, 367-374. 